Florida International University

FIU Digital Commons

7-16-2019

\title{
Prefrontal Pathways Provide Top-Down Control of Memory for Sequences of Events
}

Maanasa Jayachandran

Stephanie B. Linley

Maximilian Schlecht

Stephen V. Mahler

Robert P. Vertes

See next page for additional authors

Follow this and additional works at: https://digitalcommons.fiu.edu/psychology_fac

Part of the Medicine and Health Sciences Commons, and the Psychology Commons

This work is brought to you for free and open access by the College of Arts, Sciences \& Education at FIU Digital Commons. It has been accepted for inclusion in Department of Psychology by an authorized administrator of FIU Digital Commons. For more information, please contact dcc@fiu.edu. 


\section{Authors}

Maanasa Jayachandran, Stephanie B. Linley, Maximilian Schlecht, Stephen V. Mahler, Robert P. Vertes, and Timothy A. Allen 


\section{Cell Reports}

\section{Prefrontal Pathways Provide Top-Down Control of Memory for Sequences of Events}

\section{Graphical Abstract}

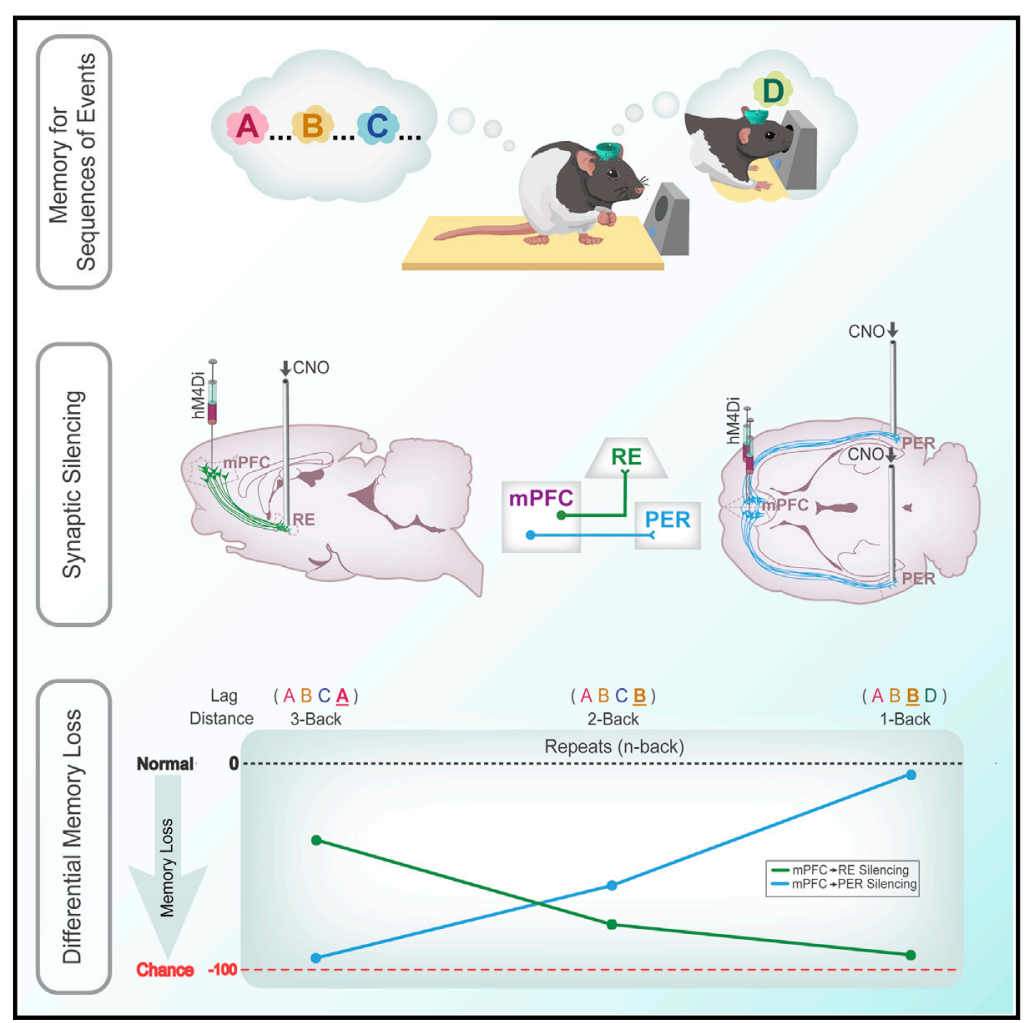

Highlights

- Non-overlapping populations of mPFC cells project to the RE and PER

- Suppressing mPFC activity impairs sequence memory

- Silencing $\mathrm{mPFC} \rightarrow \mathrm{RE}$ and $\mathrm{mPFC} \rightarrow$ PER leads to distinct deficits in sequence memory

- $\mathrm{mPFC} \rightarrow \mathrm{RE}$ and $\mathrm{mPFC} \rightarrow \mathrm{PER}$ pathways control sequence memory retrieval

\section{Authors}

Maanasa Jayachandran, Stephanie B. Linley, Maximilian Schlecht, Stephen V. Mahler, Robert P. Vertes, Timothy A. Allen

\section{Correspondence}

tallen@fiu.edu

\section{In Brief}

Jayachandran et al. demonstrate that the medial prefrontal cortex has separate projections to the nucleus reuniens of the thalamus and perirhinal cortex. The authors then demonstrate that these pathways differentially control how an episodic-like memory is retrieved. 


\title{
Prefrontal Pathways Provide Top-Down Control of Memory for Sequences of Events
}

\author{
Maanasa Jayachandran, ${ }^{1}$ Stephanie B. Linley, ${ }^{2}$ Maximilian Schlecht, ${ }^{1}$ Stephen V. Mahler, ${ }^{3}$ Robert P. Vertes, ${ }^{2}$ \\ and Timothy A. Allen ${ }^{1,4,5, *}$ \\ ${ }^{1}$ Cognitive Neuroscience Program, Department of Psychology, Florida International University, Miami, FL 33199, USA \\ ${ }^{2}$ Center for Complex Systems and Brain Sciences, Florida Atlantic University, Boca Raton, FL 33431, USA \\ ${ }^{3}$ Department of Neurobiology and Behavior, University of California, Irvine, CA 92697, USA \\ ${ }^{4}$ Department of Environmental Health Sciences, Robert Stempel College of Public Health \& Social Work, Florida International University, \\ Miami, FL 33199, USA \\ 5 Lead Contact \\ ${ }^{*}$ Correspondence: tallen@fiu.edu \\ https://doi.org/10.1016/j.celrep.2019.06.053
}

\section{SUMMARY}

We remember our lives as sequences of events, but it is unclear how these memories are controlled during retrieval. In rats, the medial prefrontal cortex (mPFC) is positioned to influence sequence memory through extensive top-down inputs to regions heavily interconnected with the hippocampus, notably the nucleus reuniens of the thalamus (RE) and perirhinal cortex (PER). Here, we used an hM4Di synapticsilencing approach to test our hypothesis that specific $\mathrm{mPFC} \rightarrow$ RE and $\mathrm{mPFC} \rightarrow$ PER projections regulate sequence memory retrieval. First, we found non-overlapping populations of MPFC cells project to RE and PER. Second, suppressing mPFC activity impaired sequence memory. Third, inhibiting $\mathrm{mPFC} \rightarrow \mathrm{RE}$ and $\mathrm{mPFC} \rightarrow$ PER pathways effectively abolished sequence memory. Finally, a sequential lag analysis showed that the $\mathrm{mPFC} \rightarrow \mathrm{RE}$ pathway contributes to a working memory retrieval strategy, whereas the $\mathrm{mPFC} \rightarrow$ PER pathway supports a temporal context memory retrieval strategy. These findings demonstrate that $\mathrm{mPFC} \rightarrow \mathrm{RE}$ and $\mathrm{mPFC} \rightarrow$ PER pathways serve as top-down mechanisms that control distinct sequence memory retrieval strategies.

\section{INTRODUCTION}

We remember our lives as sequences of events, which is at the core of episodic memory. The temporal organization of memory is thought to be useful for disambiguating memories with overlapping content and is studied using a wide array of tasks (Clayton and Dickinson, 1998; Henson, 2001; Tulving, 2002; Agster et al., 2002; Allen and Fortin, 2013; Eichenbaum, 2017a).

Neurobiologically, memory for sequences of events relies on the hippocampus (HC) (Fortin et al., 2002; Kesner et al., 2002; Allen et al., 2016) and medial prefrontal cortex (mPFC)
(Barker et al., 2007; Euston et al., 2007; Hales et al., 2009; Blumenfeld et al., 2011; Tiganj et al., 2017). Each region serves a different role in sequence memory (Hsieh and Ranganath, 2015). The HC is thought to associate information with a spatiotemporal context (Eichenbaum, 2004; Knierim et al., 2006; Knierim, 2015; Skelin et al., 2019), whereas mPFC is thought to influence the retrieval of information relevant to an action or decision (Ferbinteanu et al., 2006; Euston et al., 2012; Preston and Eichenbaum, 2013).

In rats, $\mathrm{mPFC}$ is ideally situated to influence memory retrieval through its projections to the thalamus and cortex (Sesack et al., 1989; Chiba et al., 2001; Vertes, 2002; Hoover and Vertes, 2007). mPFC may bias different sequence retrieval strategies through its top-down projection pathways. If this is the case, selective inhibition of distinct mPFC projection pathways should impair sequence memory with different effects.

The most significant mPFC projections target the nucleus reuniens of the thalamus (RE) and the perirhinal cortex (PER) because these regions are interconnected with $\mathrm{HC}$ (Eichenbaum, 2017b). The RE receives widely distributed projections from limbic sites (Vertes, 2002, 2004; McKenna and Vertes, 2004), but RE projections primarily innervate HC, parahippocampus, and prefrontal cortex (Vertes, 2006; Vertes et al., 2006). Given the absence of direct projections from mPFC to HC, RE represents the primary route as follows: $\mathrm{HC} \rightarrow \mathrm{mPFC} \rightarrow \mathrm{RE} \rightarrow$ HC (Vertes et al., 2007). Notably, RE is critical to a variety of working memory tasks that have been linked to mPFC deficits (Davoodi et al., 2009; Dolleman-van der Weel et al., 2009; Cassel et al., 2013; Hallock et al., 2013; Xu and Südhof, 2013; Griffin, 2015; Ito et al., 2015; Viena et al., 2018).

Like RE, PER is the nexus of bidirectional communication between HC and mPFC (Furtak et al., 2007). PER is critical to memory for complex stimuli (Murray et al., 2000; Bussey et al., 2002; Aggleton et al., 2010; Barker and Warburton, 2011; Feinberg et al., 2012), as well as for temporal aspects of memory (Murray and Richmond, 2001; Bussey et al., 2005; Allen et al., 2007; Barker et al., 2007; Bang and Brown, 2009; Chen et al., 2016; Naya et al., 2017).

Here, we identify the $\mathrm{mPFC} \rightarrow \mathrm{RE}$ and $\mathrm{mPFC} \rightarrow \mathrm{PER}$ projection pathways, and then test their contributions to sequence memory using a task that allows for an analysis of the underlying strategy (Allen et al., 2014, 2016). Briefly, rats sampled two sequences of odors and demonstrated sequence memory by identifying 

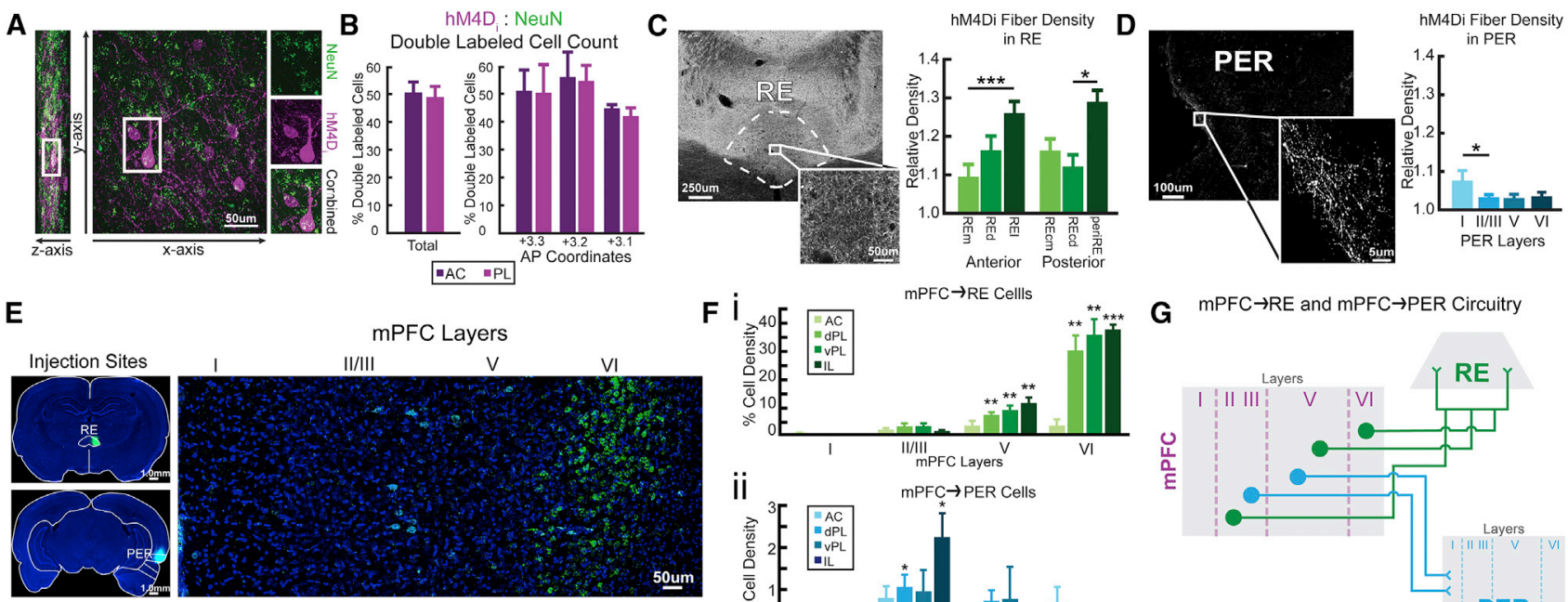

\section{$\mathbf{F}$}
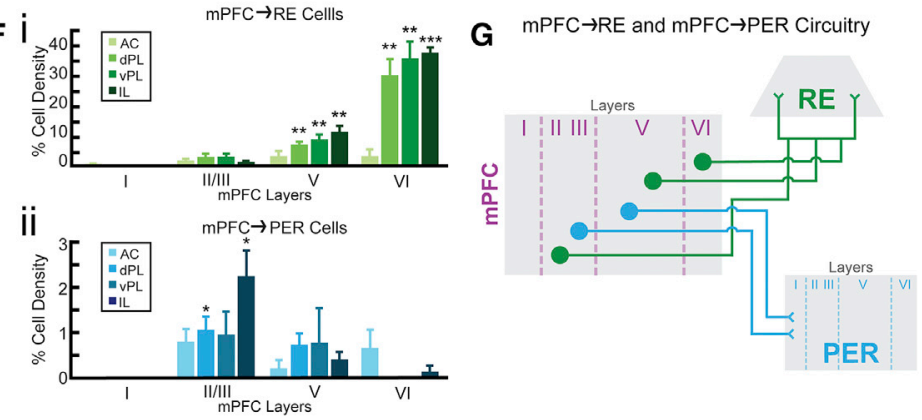

Figure 1. hM4Di Expression and Retrograde Labeling of RE and PER in mPFC

(A) hM4Di-mCherry (purple) and NeuN (neuron-specific; green) cells in mPFC.

(B) h4MDi expression rates in the prelimbic cortex (PL) and anterior cingulate cortex (AC).

(C) Axonal mPFC hM4Di expression in RE. hM4Di fiber density in RE across six subdivisions.

(D) Axonal mPFC hM4Di expression in PER across layers.

(E) RE (green) and PER (cyan; color was altered for consistency purposes) injections sites with CTB conjugated with Alexa Fluor 488 and 594 . mPFC projects to RE and PER from separate cell populations.

(F) Cell density of $\mathrm{mPFC} \rightarrow \mathrm{RE}$ (i) and $\mathrm{mPFC} \rightarrow$ PER (ii) across subregions and layers.

(G) Conceptual model of mPFC neurons with projections to RE and PER. mPFC provides direct excitatory inputs to RE and PER. $\mathrm{mPFC} \rightarrow \mathrm{RE}$ and $\mathrm{mPFC} \rightarrow \mathrm{PER}$ pathways originate from two distinct cell populations and project from specific cell layers in mPFC. $\mathrm{mPFC} \rightarrow \mathrm{RE}$ projects more toward the lateral parts of RE and $\mathrm{mPFC} \rightarrow$ PER projects to layer I of PER.

All data are represented as mean \pm SEM. ${ }^{*} \mathrm{p}<0.05 ;{ }^{* *} \mathrm{p}<0.01 ;{ }^{* * *} \mathrm{p}<0.001$

"in sequence" (InSeq) and "out of sequence" (OutSeq) odors. First we, examined mPFC projections to RE and PER using anterograde and retrograde tracers. Second, we tested the role of $\mathrm{mPFC}$ in sequence memory in rats expressing inhibitory DREADDs (designer receptor exclusively activated by designer drugs; hM4Di). Third, we tested the role of $\mathrm{mPFC} \rightarrow \mathrm{RE}$ and $\mathrm{mPFC} \rightarrow$ PER pathways using a synaptic-silencing approach (Mahler et al., 2014; Stachniak et al., 2014; Roth, 2016; Smith et al., 2016; Lichtenberg et al., 2017). Finally, we used a lag analysis across specific probes (e.g., ABA, where $A$ is a repeated item from two positions earlier, or $\bar{A} \bar{B} \underline{D}$, where $D$ skips ahead one position) to look at the unique contributions of $\mathrm{mPFC} \rightarrow \mathrm{RE}$ and $\mathrm{mPFC} \rightarrow \mathrm{PER}$ pathways. Theoretically, differences in lag performance patterns on repeated items can be used to distinguish the contributions of working memory and temporal context memory.

We found that distinct populations of mPFC cells project to RE and PER. We then show mPFC is critical to sequence memory. Silencing $\mathrm{mPFC} \rightarrow \mathrm{RE}$ and $\mathrm{mPFC} \rightarrow$ PER projections abolished sequence memory and led to a unique pattern of behavioral deficits. Silencing $\mathrm{mPFC} \rightarrow \mathrm{RE}$ produced a deficit consistent with reduced working memory, whereas silencing $\mathrm{mPFC} \rightarrow$ PER produced a deficit consistent with reduced temporal context memory (i.e., graded memory retrieval based on sequential proximity). These findings advance the concept that separate MPFC circuits target RE and PER and differentially control sequence memory.

\section{RESULTS}

\section{Incubation Time of AAV-hM4Di (Adeno-Associated} Virus) in mPFC Neurons

We targeted mPFC, which has been implicated in the temporal organization of memory (Uylings et al., 2003; Devito and Eichenbaum, 2011; Tiganj et al., 2017, 2018), using an axon-preferring hM4Di variant (AAV9.CAG.mCherry-2a-hM4D ${ }_{i}^{\text {nrxn }}$.WPRE.SV40), referred to as $h M 4 D_{i}^{\text {nrxn }}$ (neurexin; Stachniak et al., 2014). This variant exhibits enhanced axonal expression and reduced somatic expression. Activation of the $\mathrm{hM} 4 \mathrm{D}_{\mathrm{i}}^{\mathrm{nrxn}}$ variant at synaptic terminals inhibits synaptic transmission without somatic hyperpolarization (Stachniak et al., 2014). The gestation time for these experiments was determined by injecting hM4Di in a separate group of rats ( $n=4,1$ per time point) that were perfused at 1,2, 4, or 8 weeks after surgery. At 2 weeks, the virus was well expressed (Figure S1).

\section{Localization of AAV-hM4Di Transduction and Expression Patterns}

We used immunohistochemistry to visualize hM4Di expression resulting from the AAV viral constructs and found expression to be mostly restricted to the anterior cingulate and prelimbic cortices of mPFC. To estimate the localization of hM4Di, we processed slices for dual immunofluorescence in a subset of animals $(n=3)$ using antisera for mCherry and NeuN (Figure 1A). We counted mCherry-labeled cells and NeuN-labeled cells at 
three anterior-posterior (AP) levels: at the core of the injection site in layers $5 / 6$ of mPFC (AP +3.2); and anterior/posterior to this level $( \pm 120 \mu \mathrm{m})$, defining the ratio of mCherry to NeuNlabeled cells as the percentage of hM4Di-infected cells (Figure 1B). Overall, hM4Di-infected neurons in prelimbic cortex $(48.99 \% \pm 4.32 \%)$ and anterior cingulate cortex $(50.61 \% \pm$ $3.93 \%)$ did not differ $\left(t_{(16)}=0.779, p=0.447\right)$.

\section{Organization of mPFC Projections to RE and PER}

We examined the organization of mPFC projections to RE and PER using anterograde and retrograde methods. First, we mapped $\mathrm{mPFC}$ projections to RE and PER using immunolabeling of mCherry terminals taking advantage of the anterograde properties of AAV9.CAG.mCherry-2a-hM4D ${ }_{i}^{n r x n}$. Fiber expression patterns were characteristic of mPFC projection sites to the forebrain, including the orbital and insular cortices, ventral striatum, and the entire intralaminar and midline thalamus, including predominantly RE (Figure 1C), PER (Figure 1D), and entorhinal cortices, indicating good anterograde transport and allowing for clozapine $\mathrm{N}$-oxide (CNO) inhibition of mPFC terminal fibers in RE (Vertes, 2002; Hoover and Vertes, 2007, 2012; Vertes et al., 2007) and PER (Furtak et al., 2007). The density of mPFC fibers across subdivisions of each terminal site was quantified in a subset of rats $(n=3)$. mPFC fiber densities in RE and PER were tested using one-sample $t$ tests against a relative density $(R D)=1$ (the referenced area), which yielded significant differences of $p \leq 0.001$ for all regions and laminar divisions of RE and PER.

RE contained a dense plexus of hM4Di fibers that spanned the rostrocaudal extent of the nucleus. mPFC hM4Di fibers distributed strongest to the lateral aspects of $R E$ including perireuniens (periRE) (Figures $1 \mathrm{C}$ and $4 \mathrm{C}$ ). ROls for RE were taken from six separate subdivisions across the AP plane: medial (REm), dorsal (REd), and lateral (REI) divisions rostrally and medial (REcm, dorsal [REcd], and periRE laterally in caudal RE). Across anterior RE, there was a significant difference in the distribution of mCherrylabeled fibers $\left(F_{(2,16)}=8.81, p=0.003\right)$. hM4Di mPFC axons targeted REd $(R D=1.166 \pm 0.078)$ and $R E I(R D=1.264 \pm 0.103)$ with the heaviest of fiber innervation in the lateral "wings" (Figures 1C and 4C). By comparison, the density of mPFC axons in REm $(R D=1.095 \pm 0.480)$ was substantially less than REI $(p=0.001)$ and REd $(p=0.054)$. There was a similar pattern of innervation across RE for the posterior aspect of RE, with significant differences in density across subdivisions mediolaterally $\left(F_{(2,12)}=4.334, p=0.038\right)$ with intense fiber labeling in periRE $(R D=1.238 \pm 0.100)$, followed by $R E d(R D=1.161 \pm 0.063)$, and fewer fibers covering the medial $R E c p(R D=1.132 \pm 0.051)$.

In PER, the density of mPFC fibers differed significantly across layers of $P E R\left(F_{(3,24)}=7.025, p=0.014\right)$, signifying a distinct laminar organization in PER. hM4Di mPFC axons terminated densely in layer I, with significant differences $(R D=1.075 \pm 0.06)$ compared to a paucity of fibers in layers II/III $(R D=1.033 \pm 0.025$; Figures $1 \mathrm{D}$ and $5 \mathrm{C})$. There were no differences in hM4Di fiber density across layers II/III, V $(\mathrm{RD}=1.029 \pm 0.017)$, and $\mathrm{VI}(\mathrm{RD}=1.035 \pm 0.019)$.

\section{Separate Populations of mPFC Neurons Project to RE and PER}

Next, we tested whether the same cells in MPFC project to both $\mathrm{RE}$ and PER, or whether these are separate cell populations.
Rats $(n=4)$ underwent surgery for a dual retrograde fluorescence labeling of cholera toxin subunit B green (CTB-488) and red (CTB-594) in RE and PER. We analyzed cells in the anterior cingulate cortex, prelimbic cortex, and infralimbic cortex across layers for retrograde labeling from either $\mathrm{RE}$ and $\mathrm{PER} . \mathrm{mPFC} \rightarrow$ $\mathrm{RE}$ projecting cells were found in layers II/III, V, and VI (Figures $1 \mathrm{E}$ and S2A). These projections were found throughout dorsal and ventral prelimbic cortex, and infralimbic cortex, predominantly in layers $\mathrm{V}$ (Figure 1Fi; dPL, $\mathrm{t}_{(3)}=7.686, \mathrm{p}=0.005$; $\mathrm{vPL}, \mathrm{t}_{(3)}=$ 4.909, $\left.\mathrm{p}=0.016 ; \mathrm{IL}, \mathrm{t}_{(3)}=5.603, \mathrm{p}=0.011\right)$ and $\mathrm{VI}\left(\mathrm{dPL}, \mathrm{t}_{(3)}=\right.$ 5.508, $\mathrm{p}=0.012 ; \mathrm{vPL}, \mathrm{t}_{(3)}=6.210, \mathrm{p}=0.008 ; \mathrm{IL}, \mathrm{t}_{(3)}=21.840$, $\left.p=2.100 \times 10^{-4}\right)$. Only a few cells were found in anterior cingulate cortex. $\mathrm{mPFC} \rightarrow \mathrm{PER}$ projecting cells were observed throughout layers II/III and V, with the highest density in layer II/III (Figure 1Fii; dPL, $\mathrm{t}_{(3)}=3.654, \mathrm{p}=0.035$; IL, $\mathrm{t}_{(3)}=3.849, \mathrm{p}=$ 0.031), consistent with previous literature (Hwang et al., 2018; Mathiasen et al., 2019). We did not find labeled cells in layer I. Moreover, we did not find cells in mPFC that projected to both RE and PER (no dual-labeled cells), suggesting no overlap. To identify $\mathrm{mPFC}$ projection cell types, a set of tissue was processed using the antibody for glutamic acid decarboxylase (GAD67). We did not find any dual-labeled cells in mPFC for GAD67 and CTB-488/594. This confirmed both populations of mPFC cells are excitatory. Notably, RE is populated exclusively by excitatory glutamatergic cells and presumably mPFC cells synapse onto these neurons (Vertes et al., 2007). In PER, mPFC axons synapse onto pyramidal cells (Hwang et al., 2018). Overall, these results show that separate populations of excitatory $\mathrm{mPFC}$ neurons, originating from prelimbic and infralimbic cortex, project to RE and PER (Figure 1G).

\section{Sequence Memory Task and Overall Performance}

We trained rats in an odor sequence task (Allen et al., 2016). The behavioral setup was automated and allows for repeated delivery of distinct odors (pure chemical odorants) between two odor ports. We used two sequences, each with four odors that could be delivered at opposite ends of a straight alley (Figure 2A). The use of two sequences eliminated the possibility rats could remember a single sequence throughout the testing period and increased the overall memory load. Furthermore, the use of two sequences allowed us to tease apart different retrieval strategies as rats had to repeatedly retrieve sequences from long-term memory stores. To obtain a water reward, rats had to demonstrate sequence memory by indicating whether the odors were InSeq (holding the nose-poke response $>1 \mathrm{~s}$ ) or OutSeq (withdrawing from the nose port $<1 \mathrm{~s}$ ) within a sequence (Figures 2B and 2C). We calculated a sequence memory index (SMI) to measure overall sequence memory while controlling for individual differences in poke-hold behavior (Allen et al., 2014). The SMI normalizes the proportion of InSeq and OutSeq items presented during a single session for comparison across sessions and scores sequence memory with a value ranging from -1 to 1 . A score of 0 indicates chance performance. A score of 1 indicates perfect performance. Rats were trained in the sequence memory task in progressive stages over several weeks as depicted in Figure 2D. After reaching behavioral criterion (asymptotic sequence memory performance levels over multiple sessions), the rats underwent surgery for bilateral microinjection into mPFC of one of two viral groups 
A

\section{Sequence $1 \longmapsto$ Alternate Between Sequences}

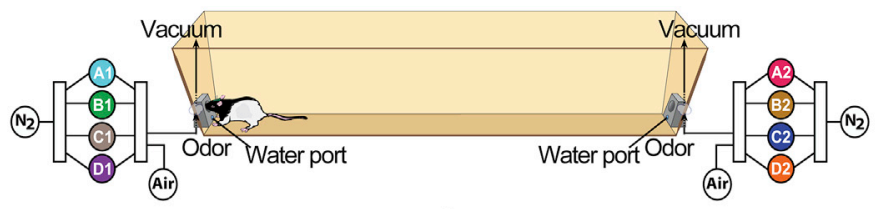

B

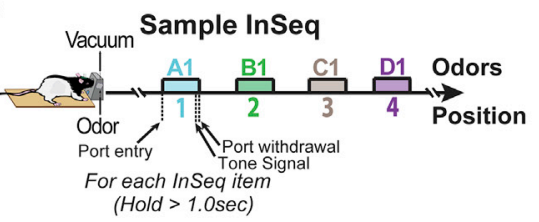

C

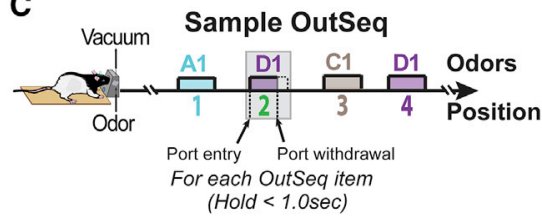

D

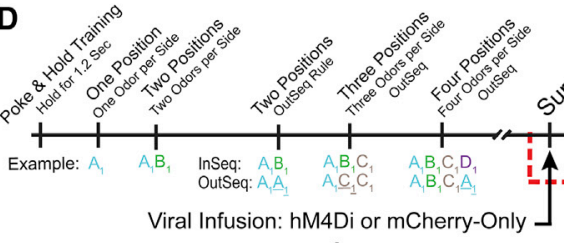

Cannula in RE and PER (Bilateral)

E

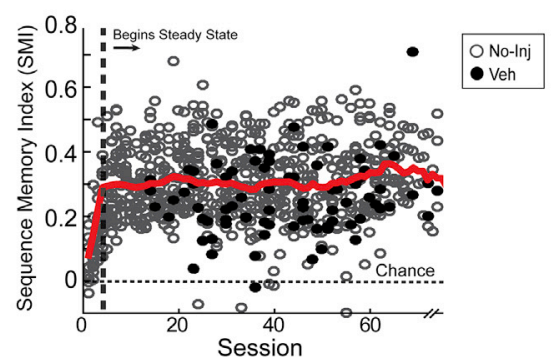

$\mathbf{F}$

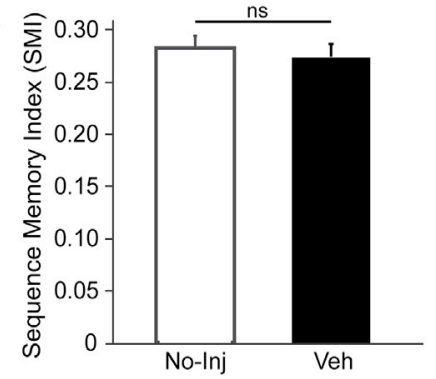

Figure 2. Sequence Memory Task

(A) A linear track was used with odor ports at each end where two separate four-odor sequences $\left(A_{1}, B_{1}, C_{1}, D_{1}\right.$, or $\left.A_{2}, B_{2}, C_{2}, D_{2}\right)$ were presented. ( $B$ and $C$ ) Rats had to correctly identify the odor as either InSeq $(70 \%$ of the time; B) or OutSeq $(30 \%$ of the time; C).

(D) After reaching steady-state performance, we focused on two experimental blocks: (1) i.p. injection suppressing MPFC neurons and (2) intracerebral infusions targeting mPFC terminals in RE or PER. The boxes represent a sample schedule. Veh days are denoted in black, and No-Inj days are in white. (E) We used the sequence memory index (SMI) as a summary measure. The red line represents the mean SMI of both No-Inj and Veh sessions (sliding window of 10 sessions).

(F) SMI was not significantly different between the No-Inj and Veh sessions.

All data are represented as mean \pm SEM. ns, not significant.

0.008), with no significant difference between sequences $\left(t_{(704)}=1.279, p=\right.$ 0.201 ), indicating rats successfully switched between the two sequences. Additionally, performance on either of the sequences did not differ significantly on No-Inj and Veh days (Figure S4A; sequence 1: $t_{(52)}=-1.237, p=0.222$; sequence $\left.2: t_{(52)}=0.082, p=0.935\right)$.

\section{Suppressing mPFC Activity Impairs Sequence Memory}

Evidence suggests the mPFC makes essential contributions to sequence mem-

(hM4Di': AAV9.CAG.mCherry-2a-hM4Dinrxn.WPRE.SV40; or mCherry-only: AAV9.CB7.CI.mCherry.WPRE.rBG). Chronic guide cannulas were implanted targeting RE and PER bilaterally. After recovery, the rats continued performing the sequence task daily and the experimental manipulations (intraperitoneal [i.p.] injections and intracerebral infusions) were started $\sim 3$ weeks later.

Overall, the rats demonstrated strong sequence memory (Figure 2E; $\mathrm{SMI}_{\text {well-trained: }} 0.302 \pm 0.034$ ) and performance did not differ significantly between no-injection/no-intracerebral infusion (No-Inj) and vehicle injection/vehicle infusion (Veh) days (Figure $2 \mathrm{~F} ; \mathrm{t}_{(90)}=0.426, \mathrm{p}=0.671$ ). Expected versus observed frequencies were analyzed with $G$ tests to determine whether the observed frequency of InSeq and OutSeq responses for a given session significantly differed from chance. Single-subject analyses revealed every rat differentiated InSeq and OutSeq items at levels well above chance (Figure S3; all G tests had $\mathrm{p}$ values $<0.05)$. Moreover, the rats performed well on position 2 (SMI $\left._{\text {Pos } 2}: 0.282 \pm 0.008\right)$, position $3\left(\mathrm{SMI}_{\mathrm{Pos} 3}: 0.321 \pm 0.009\right)$, and position $4\left(\mathrm{SMI}_{\mathrm{Pos} 4}: 0.282 \pm 0.018\right)$, indicating memory for the entire length of each sequence. Position 1 was excluded because an OutSeq item was never presented in that position. Rats also performed well above chance levels on sequence 1 $\left(\mathrm{SMI}_{\text {Seq1 }}: 0.305 \pm 0.007\right)$ and sequence $2\left(\mathrm{SMI}_{\text {Seq2 }}: 0.292 \pm\right.$ ory (Devito and Eichenbaum, 2011). Here, we examined the contribution of mPFC to sequence memory via systemic CNO (i.p., $1 \mathrm{mg} / \mathrm{kg}$ ) or Veh injections (Figure 3A). The spread of $\mathrm{hM} \mathrm{Di}^{+}$neurons across mPFC was precisely mapped. hM4Di cells were concentrated in the rostral- to mid-levels of the prelimbic and the anterior cingulate cortex, with dense labeling in the deep cortical layers (Figure $3 \mathrm{~B}$ ). While mCherry-only cells were present throughout the rostral caudal extent of the prelimbic cortex, $\mathrm{hM} 4 \mathrm{Di}^{+}$expression was limited to the rostral aspects of the anterior cingulate cortex, with only a small number of infected cells in the posterior and ventral divisions of the anterior cingulate cortex. In some $\mathrm{hM}_{4} \mathrm{Di}^{+}$cases, very small numbers of labeled cells extended into the medial orbital, ventral orbital, infralimbic, medial agranular cortex, and claustrum. The spread of mCherry cells was mapped in the mCherry-only control rats (Figure S5A).

We considered each corresponding Veh and CNO injection as a repeated condition across behavioral sessions. Suppression of mPFC neurons with CNO in the hM4Di+ group (SMI: $0.056 \pm$ 0.121 ) significantly reduced SMI scores compared with Veh injection (SMI: $0.254 \pm 0.062$ ) the first time we ran this condition (Figures 3C and 3D; first: $\mathrm{t}_{(12)}=5.200, \mathrm{p}=2.210 \times 10^{-4}$, Cohen's $d=1.449$ ), but in the second and third repeats of this condition, 
A

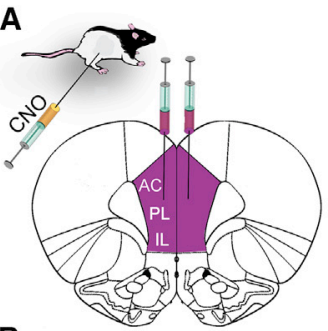

B

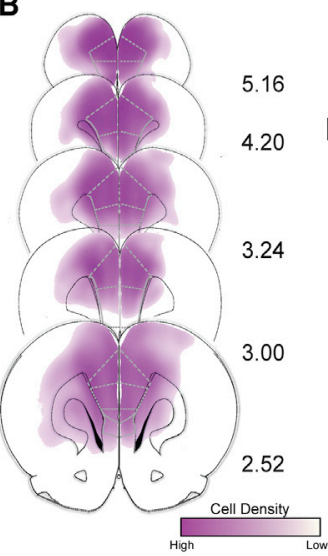

C

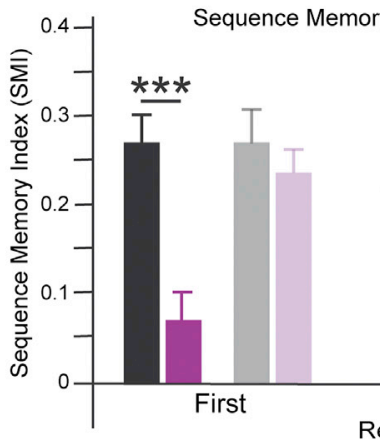

\section{D}

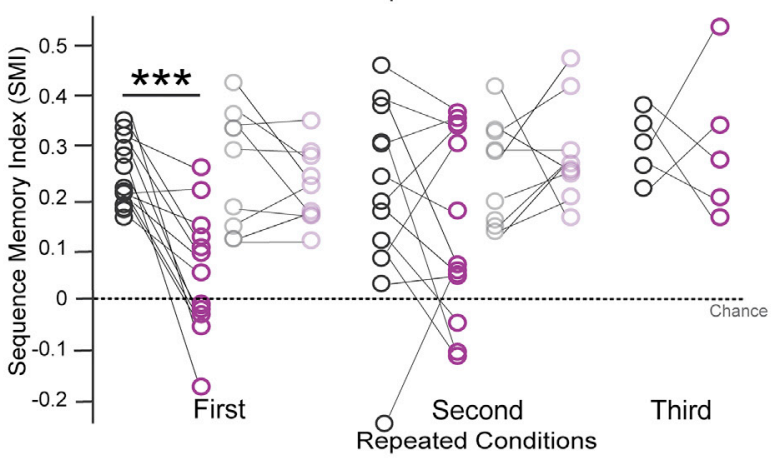

E

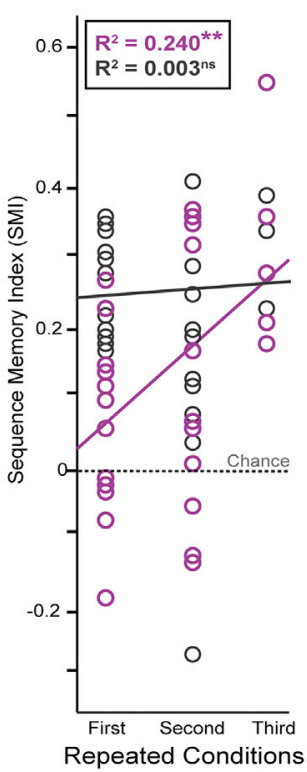

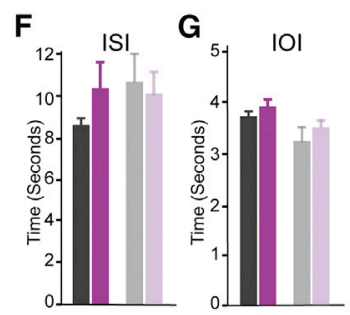
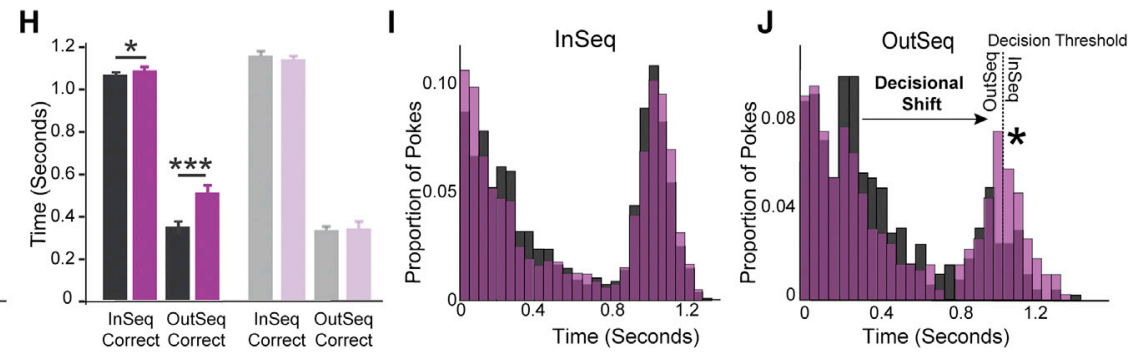

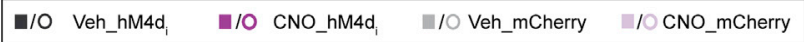

Figure 3. mPFC Cortex Is Needed for Sequence Memory

(A) AAV9.hM4Di was injected bilaterally into mPFC.

(B) Representation of AAV9.hM4Di viral spread in mPFC for all rats $(n=13)$.

(C) Performance differed between $\mathrm{hM}_{4} \mathrm{Di}^{+}$animals injected with Veh and CNO in the first repeated condition, but not the second and third repeated conditions.

No differences between Veh and CNO were detected in the mCherry-only group.

(D) Individual rat performance for each repeated condition in both groups.

(E) In the hM4Di+ group, there was a positive linear relationship across repeated conditions after CNO injections, but not after Veh injections.

(F) ISI was not significantly different between the Veh and CNO conditions in either group.

(G) IOI was not significantly different between the Veh and CNO conditions in either group.

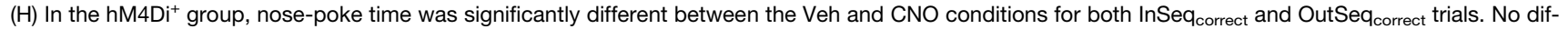
ferences between Veh and CNO were detected in the mCherry-only group.

(l) $\mathrm{hM} 4 \mathrm{Di}^{+}$group poke times show only subtle shifts in behavior.

$(J) \mathrm{hM}_{4} \mathrm{Di}^{+}$group poke times show a decisional shift between the Veh and CNO conditions with more OutSeq trials incorrectly identified as InSeq.

All data are represented as mean \pm SEM. ${ }^{\star} p<0.05 ;{ }^{\star \star} p<0.01 ;{ }^{* \star *} p<0.001 ;$ ns, not significant.

we observed no significant effect (second: $t_{(12)}=1.039$, $\mathrm{p}=0.319$; third: $\mathrm{t}_{(4)}=0.031, \mathrm{p}=0.977$ ). Overall, we performed three repeated conditions for the first cohort of $\mathrm{hM} 4 \mathrm{Di}^{+}$rats $(n=5)$. For the second cohort of $h M 4 \mathrm{Di}^{+}$rats $(n=8)$ and mCherry-only rats $(n=9)$, we decreased the number of repeated conditions to two, because the third repeated condition with CNO administration had no effect. We found a significant interaction between the injection and repeated condition $\left(F_{(1,12)}=5.410, p=0.038\right)$. Furthermore, a linear regression was performed to determine the relationship between CNO administration and repeated conditions. Figure $3 \mathrm{E}$ shows there was a moderate positive linear relationship between the repeated conditions and $\mathrm{CNO}$ in the $\mathrm{hM} 4 \mathrm{Di}^{+}$group (Pearson's $\left.r=0.490, R^{2}=0.240, p=0.003\right)$, but no relationship between 
the repeated condition and Veh in the $\mathrm{hM} 4 \mathrm{Di}^{+}$group (Pearson's $r=0.057, R^{2}=0.003, p=0.380$ ). An ANOVA yielded similar results, revealing a significant relationship between repeated conditions and SMI for CNO injections $\left(F_{(1,29)}=9.167, p=0.005\right)$. Importantly, CNO administration had no significant effects in the mCherry-only group (Figures $3 C$ and $3 D$; first: $t_{(8)}=1.208$, $p=0.262$; second: $t_{(8)}=-0.623, p=0.551$ ), indicating the effects observed in the hM4Di group were specific to hM4Di receptor activity on mPFC neurons. While infected cells in mCherry-only rats were visualized outside of mPFC, extending to the orbital and motor cortices, the relative density and pattern of labeling of terminal fibers to target sites (RE and PER) was similar to $\mathrm{hM} 4 \mathrm{Di}^{+}$rats. Furthermore, the lack of behavioral effects on these rats confirmed our findings were not associated with non-specific effects related to the viral construct or CNO. mCherry expression in mPFC from a representative case of $\mathrm{hM}^{2} \mathrm{Di}^{+}$and mCherry-only is depicted in Figures S5D and S5F. While neuronal labeling was virtually restricted to the injection sites, sporadic cells (less than $\sim 0.1 \%-1 \%$ ) were observed at the target sites, most likely associated with weak retrograde transport of adenovirus constructs (Castle et al., 2014; Tervo et al., 2016) as reported for other hM4Di applications (DiBenedictis et al., 2015). Finally, sequence memory performance levels were similar between sequence 1 and sequence 2 during systemic $\mathrm{CNO}$ and Veh administration, with no significant differences between them (Figure S4B; first: Veh, $t_{(11)}=-1.023, p=0.328$, CNO, $t_{(11)}=-1.231, p=0.244$; second: Veh, $t_{(9)}=2.086$, $\left.\mathrm{p}=0.067, \mathrm{CNO}, \mathrm{t}_{(10)}=0.533, \mathrm{p}=0.606\right)$, suggesting a general sequence memory deficit.

Next, we examined the possibility hM4Di suppression of mPFC activity produced non-mnemonic effects relevant to the sequence task. To test for this possibility, we measured the time it took the rats to run between sequences (inter-sequence interval [ISI]), the time spent between each odor trial (inter-odor interval [IOI]), and nose-poke times. The ISI did not differ significantly between the Veh and CNO conditions in either the $\mathrm{hM} \mathrm{Di}^{+}$or mCherry-only groups (Figure 3F; hM4Di': $\mathrm{t}_{(25)}=$ $-1.427, p=0.166$; mCherry-only: $\left.t_{(17)}=0.454, p=0.655\right)$, suggesting rats in both groups ran at similar rates between sequences. Furthermore, we found no effects on the IOI (Figure 3G; $h M 4 \mathrm{Di}^{+}: \mathrm{t}_{(25)}=-1.616, \mathrm{p}=0.119 ;$ mCherry-only: $\mathrm{t}_{(17)}=-1.056$, $p=0.306$ ), suggesting rats collected water rewards and engaged odors at similar rates. Evaluation of a potential holding bias during the task comparing the Veh and CNO conditions revealed no significant difference between holding $\left(t_{(12)}=-0.875\right.$, $\mathrm{p}=0.399)$ and not holding $\left(\mathrm{t}_{(12)}=0.506, \mathrm{p}=0.622\right)$. We also evaluated whether CNO affected nose-poke times on InSeq

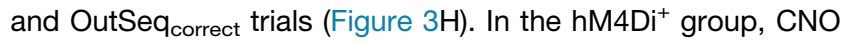
suppression of mPFC activity significantly increased nosepoke times in both $\operatorname{InSeq}_{\text {correct }}$ and OutSeq ${ }_{\text {correct }}$ trials $\left(\mathrm{hM} \mathrm{Di}^{+}\right.$: InSeq correct, $\mathrm{t}_{(25)}=-2.672, \mathrm{p}=0.013$, OutSeq correct, $\mathrm{t}_{(25)}=$ $-3.944, p=0.001$ ). No significant differences were detected in the mCherry-only group (mCherry-only: InSeqcorrect, $t_{(17)}=$ $0.973, p=0.344$, OutSeq $\left.{ }_{\text {correct }}, t_{(17)}=-0.186, p=0.853\right)$, indicating i.p. CNO did not affect nose-poke behavior in the task. The increase in hold times in the hM4Di+ group may indicate uncertainty regarding whether a trial was InSeq or OutSeq and thus a decisional shift rather than a deficit related to basic nose-poke and hold behavior. To further evaluate this possibility, we examined nose-poke distributions for both InSeq and OutSeq trials (Figures $3 \mathrm{I}$ and $3 \mathrm{~J}$ ). The InSeq distribution shows the proportion of nose pokes in the $\mathrm{hM} 4 \mathrm{Di}^{+}$group remained similar between the Veh and CNO conditions, with only modest differences. On OutSeq trials, however, there was a clear shift in the proportion of trials near the 1-s decision threshold, suggesting a shift toward making InSeq decisions. Thus, the nose-poke differences indicate a decisional shift in the $\mathrm{hM} 4 \mathrm{Di}^{+}$group following CNO administration. Overall, these results demonstrate suppression of mPFC neurons by systemic CNO administration in the hM4Di ${ }^{+}$ group impaired memory for sequences of events, but the effect diminished with subsequent administrations of CNO.

\section{Synaptic Silencing of $\mathrm{mPFC} \rightarrow$ RE Projections Abolished Sequence Memory}

Our primary goal was to examine whether mPFC inputs to RE and PER, structures heavily interconnected with the $\mathrm{HC}$, contribute to sequence memory (Eichenbaum, 2017b). We tested top-down mPFC inputs using intracerebral CNO infusions $(1 \mu \mathrm{L}$ at $1 \mu \mathrm{g} / \mu \mathrm{L}$ per cannula) targeting RE and PER (within subject) on different days. The daily schedule for RE and PER infusions was randomized and counterbalanced (Figure 1F) across rats and repeated conditions to avoid an order effect.

We first examined $\mathrm{mPFC} \rightarrow \mathrm{RE}$ projections (Figure $4 \mathrm{~A}$ ). Cannula placement for intracerebral infusions was confirmed with cresyl violet (Figures 4B, S5B, S5E, and S5G). Silencing mPFC terminals in $\mathrm{RE}$ in the $\mathrm{hM} 4 \mathrm{Di}^{+}$group significantly impaired sequence memory (Figures 4D and 4E). The effects of CNO infusion clearly differed from Veh infusion $\left(F_{(1,9)}=130.850\right.$, $\left.p=1.000 \times 10^{-6}\right)$, with no differences across repeated conditions (Figures $4 \mathrm{D}$ and $\left.4 \mathrm{E} ; \mathrm{F}_{(2,18)}=1.012, \mathrm{p}=0.366\right)$. The interaction between factors was not significant $\left(F_{(2,18)}=0.914\right.$, $p=0.388$ ). Thus, silencing $\mathrm{mPFC} \rightarrow \mathrm{RE}$ synapses powerfully and repeatedly abolished sequence memory. Performance of the mCherry-only group showed no significant difference between $\mathrm{CNO}$ and Veh conditions or across repeated conditions (Figures 4D and 4E; infusion: $F_{(1,8)}=0.492, p=0.503$; repeated conditions: $F_{(2,16)}=0.025, p=0.967$; infusion $\times$ repeated conditions: $\left.F_{(2,16)}=1.931, p=0.184\right)$, controlling for non-specific CNO effects in RE. A linear regression was performed to investigate the relationship between SMI (CNO and Veh) and repeated conditions for the hM4Di+ group, which was not significant (Figure 4F; CNO: Pearson's $r=0.101, R^{2}=0.010, p=0.307$; Veh: Pearson's $\left.r=0.259, R^{2}=0.067, p=0.096\right)$. In the $\mathrm{hM} 4 \mathrm{Di}^{+}$group, sequence 1 and sequence 2 were similar across all repeated conditions (Figure S4C; first: Veh, $\mathrm{t}_{(9)}=0.989, \mathrm{p}=0.348, \mathrm{CNO}$, $\mathrm{t}_{(9)}=-1.665, \mathrm{p}=0.130$; second: Veh, $\mathrm{t}_{(9)}=0.242, \mathrm{p}=0.814$, CNO, $t_{(9)}=0.322, p=0.754$, third: Veh, $t_{(9)}=-0.506$, $\left.\mathrm{p}=0.625, \mathrm{CNO}, \mathrm{t}_{(9)}=1.069, \mathrm{p}=0.313\right)$, indicating a general sequence memory deficit.

We looked at the non-mnemonic effects of $\mathrm{mPFC} \rightarrow \mathrm{RE}$ silencing by examining ISI, IOI, and nose-poke behavior. In both the hM4Di ${ }^{+}$and mCherry-only groups, the ISI and IOI were not significantly different between Veh and CNO conditions (Figures 4G and 4H; hM4Di': ISI, $\mathrm{t}_{(29)}=0.502, \mathrm{p}=0.619$, IOI, $\mathrm{t}_{(29)}=0.394, \mathrm{p}=0.696 ;$ mCherry: ISI, $\mathrm{t}_{(26)}=-1.123, \mathrm{p}=0.272$, IOI, $\left.\mathrm{t}_{(26)}=1.119, \mathrm{p}=0.273\right)$. The holding bias during the task 


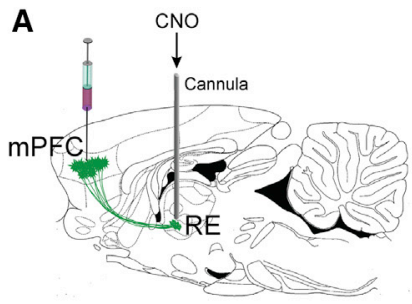

B

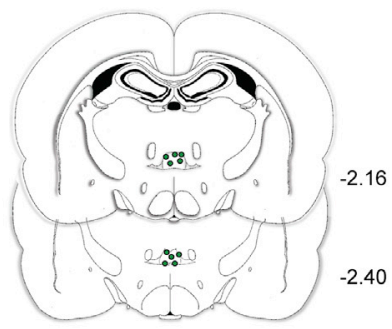

C

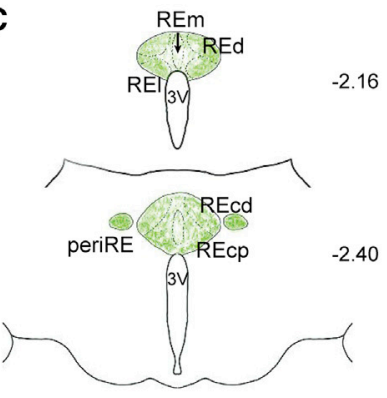

D

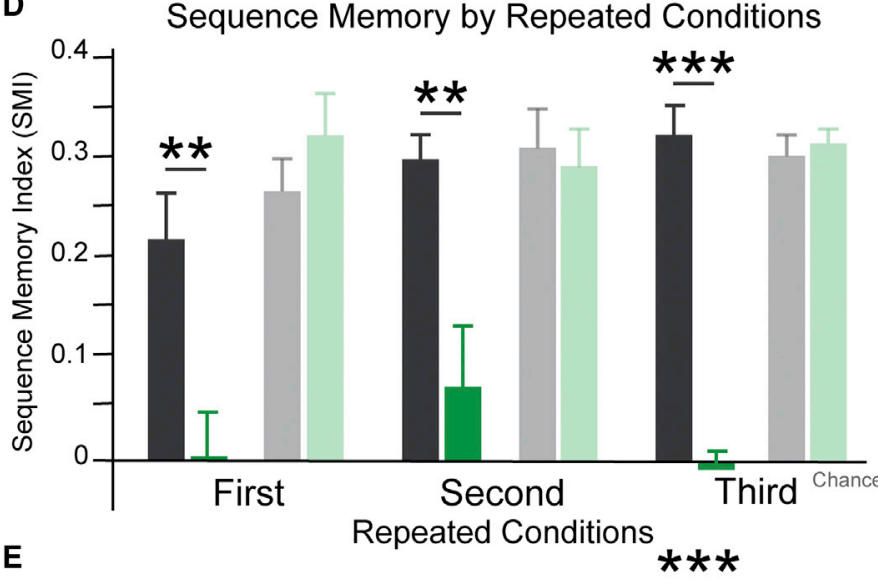

$\mathbf{F}$

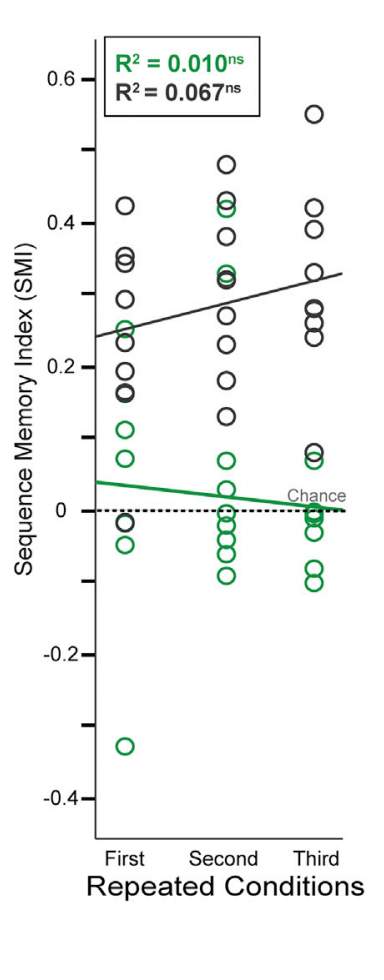

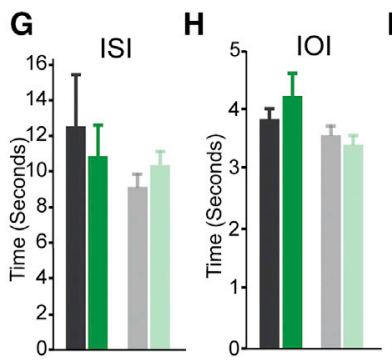

I

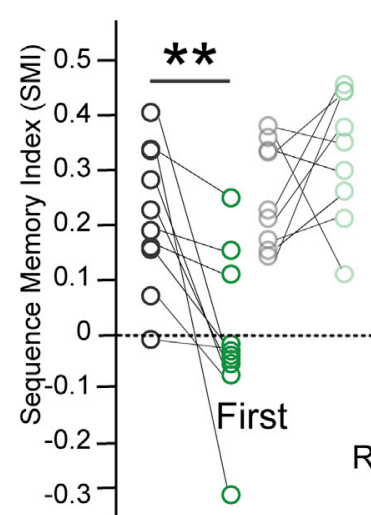

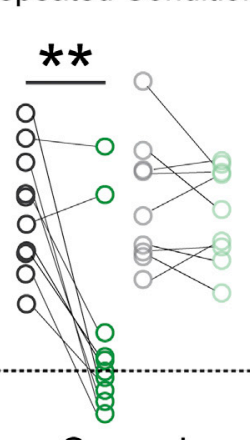

Second Repeated Conditions

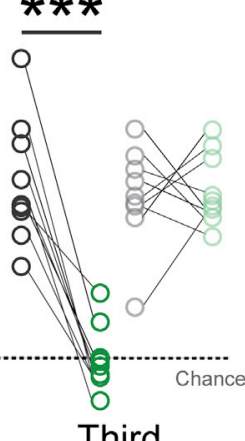

Third
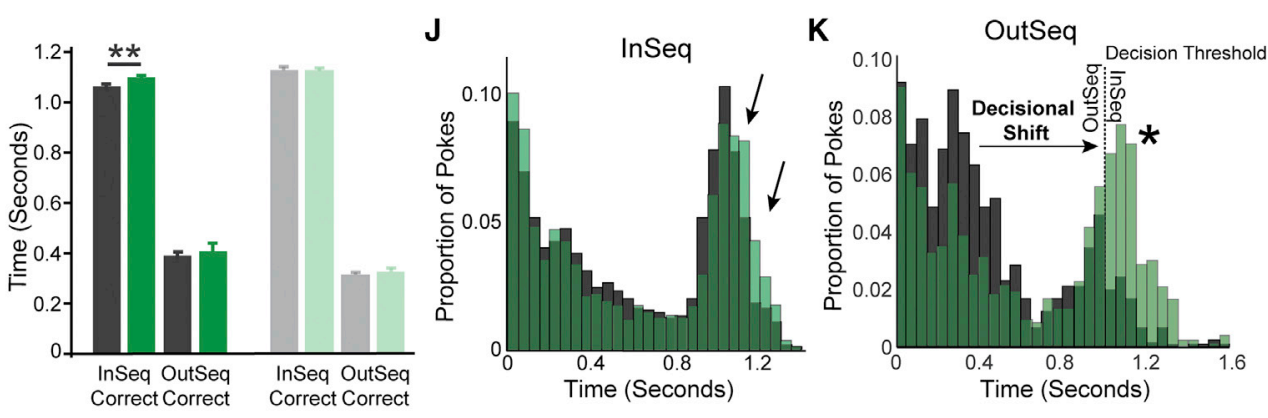

ם/O Veh_hM4d,

-10 CNO_hM4d

[1O Veh_mCherry

¿/OCNO_mCherry

Figure 4. Synaptic Silencing of $\mathrm{mPFC} \rightarrow$ RE Pathway Abolished Sequence Memory

(A) Guide cannulas targeted RE.

(B) Locations of RE infusion cannulas in all rats $(n=10)$.

(C) Schematic representation of mPFC hM4Di fiber density in RE.

(D) In the $\mathrm{hM} \mathrm{Di}^{+}$group, SMI was significantly different between Veh and CNO conditions in all three repeated conditions. There were no effects in the mCherry-only group.

(E) Individual rat performances for each repeated condition in both groups.

(F) In the hM4Di+ group, there was no significant relationship between repeated conditions and infusions.

(G) ISI was not significantly different between RE Veh and CNO infusions in either group.

(H) IOI was not significantly different between RE Veh and CNO infusions in either group.

(I) In the hM4Di+ group, InSeq ${ }_{\text {correct }}$ nose-poke times were significantly different between Veh and CNO. OutSeq significantly between the Veh and CNO conditions. No differences between Veh and CNO were detected in the mCherry-only group.

(J) hM4Di ${ }^{+}$group poke times were relatively similar.

(K) hM4Di+ group nose-poke times show a decisional shift (indicated by a star) between the Veh and CNO conditions toward more OutSeq odors incorrectly identified as InSeq.

All data are represented as mean \pm SEM. ${ }^{*} \mathrm{p}<0.05 ;{ }^{\star \star} \mathrm{p}<0.01 ;{ }^{\star \star *} \mathrm{p}<0.001$; ns, not significant. 
did not differ significantly between the Veh and CNO conditions (holding: $\mathrm{t}_{(29)}=-0.639, \mathrm{p}=0.528$; not holding: $\mathrm{t}_{(29)}=-0.056$, $p=0.956)$. The nose-poke times were mostly similar between Veh and CNO conditions in both the hM4Di ${ }^{+}$and mCherry-only groups following RE infusions (Figure 4l; hM4Di': OutSeq ${ }_{\text {correct }}$, $\mathrm{t}_{(29)}=-0.709, \mathrm{p}=0.484$; mCherry-only: InSeq $q_{\text {correct }}, \mathrm{t}_{(26)}=$ $-0.020, p=0.984$, OutSeq $\left.{ }_{\text {correct }}, t_{(26)}=-0.777, p=0.444\right)$, but there was a slight and significant increase in the amount of time rats in the $\mathrm{hM} 4 \mathrm{Di}^{+}$group held the nose-poke response in the CNO condition on InSeq trials (InSeq correct: $t_{(29)}=-2.760$, $p=0.010)$. We further analyzed poke distributions in the $\mathrm{hM} 4 \mathrm{Di}^{+}$group for all InSeq and OutSeq trials (Figures $4 \mathrm{~J}$ and 4K). The InSeq distribution showed the proportion of nose-pokes remained similar between the Veh and CNO conditions in the $\mathrm{hM}_{4} \mathrm{Di}^{+}$group, with a slight increase in holding (Figure 4J). As shown in Figure $4 \mathrm{~K}$, CNO infusion in the $\mathrm{hM}_{4} \mathrm{Di}^{+}$group decreased the proportion of OutSeq nose-pokes near the short distribution peak ( 0.35-0.6 s) and increased the proportion near the 1-s decision threshold compared with Veh infusion. The nose-poke differences indicate a decisional shift in the $\mathrm{hM} 4 \mathrm{Di}^{+}$group following CNO infusions. Overall, these results provide strong evidence that silencing $\mathrm{mPFC} \rightarrow \mathrm{RE}$ leads to inaccurate decisions, not a basic deficit in poke and hold behavior, reflecting deficits in sequence memory.

\section{Synaptic Silencing of $\mathrm{mPFC} \rightarrow$ PER Projections} Abolished Sequence Memory

We next examined $\mathrm{mPFC} \rightarrow$ PER projections (Figure $5 \mathrm{~A}$ ). Cannula placement for intracerebral infusions was confirmed with cresyl violet (Figures 5B, S5C, S5E, and S5G). Silencing $\mathrm{mPFC} \rightarrow$ PER projections significantly and consistently impaired sequence memory across repeated conditions in the $\mathrm{hM} 4 \mathrm{Di}^{+}$ group (Figures 5D and 5E; infusion: $F_{(1,8)}=62.750, p=4.700 \times$ $10^{-5}$; repeated condition: $F_{(2,16)}=1.466, p=0.263$; infusion $x$ repeated condition: $\left.F_{(2,16)}=0.118, p=0.846\right)$. Linear regression analysis showed no significant relationship between repeated conditions and SMI following PER infusions with either CNO or Veh in the hM4Di ${ }^{+}$group (Figure 5F; CNO: Pearson's $r=0.053$, $R^{2}=0.003, p=0.399 ;$ Veh: Pearson's $r=0.167, R^{2}=0.028$, $p=0.202)$. Furthermore, CNO infusion had no significant effect on sequence memory in the mCherry-only group compared with Veh infusion (Figures 5D and 5E; infusion: $F_{(1,8)}=0.690$, $p=0.430$; repeated condition: $F_{(2,16)}=0.092, p=0.886$; infusion $x$ repeated condition: $\left.F_{(2,16)}=1.056, p=0.351\right)$. Additionally, no significant difference was detected between the two sequences under any condition (Figure S4D; first: $V$ eh, $\mathrm{t}_{(9)}=0.013, \mathrm{p}=0.990$, CNO, $\mathrm{t}_{(8)}=-0.928, \mathrm{p}=0.380$; second: Veh, $\mathrm{t}_{(9)}=-0.169$, $\mathrm{p}=0.870, \mathrm{CNO}, \mathrm{t}_{(9)}=-1.101, \mathrm{p}=0.300$; third: Veh, $\mathrm{t}_{(9)}=$ $\left.1.003, p=0.342, \mathrm{CNO}, \mathrm{t}_{(9)}=0.630, \mathrm{p}=0.545\right)$, thus indicating a general sequence memory deficit.

We evaluated non-mnemonic effects of $\mathrm{mPFC} \rightarrow \mathrm{PER}$ silencing by examining the ISI, IOI, and nose-poke behavior. In both the $\mathrm{hM}_{4} \mathrm{Di}^{+}$and mCherry-only groups, the ISI and IOI did not differ significantly between PER infusions of Veh and CNO (Figures $5 \mathrm{G}$ and $5 \mathrm{H}$; hM4 $\mathrm{Di}^{+}$: ISI, $\mathrm{t}_{(29)}=0.634, \mathrm{p}=0.531, \mathrm{IOI}$, $\mathrm{t}_{(29)}=0.456, \mathrm{p}=0.652$; mCherry: ISI, $\mathrm{t}_{(26)}=0.082, \mathrm{p}=0.935$, $\left.\mathrm{IOI}, \mathrm{t}_{(26)}=-0.874, \mathrm{p}=0.390\right)$. Furthermore, the holding bias during the task did not differ significantly between Veh and CNO conditions (holding: $\mathrm{t}_{(29)}=0.322, \mathrm{p}=0.750$; not holding: $\mathrm{t}_{(29)}=$ 1.595, $\mathrm{p}=0.122$ ). Additionally, nose-poke times in the $\mathrm{hM} 4 \mathrm{Di}^{+}$ and mCherry-only groups were similar between PER infusions of Veh and CNO (Figure 5l; hM4Di: InSeq Correct: $_{(29)}=0.621$, $\mathrm{p}=0.540, \mathrm{hM} 4 \mathrm{Di}^{+}:$OutSeq ${ }_{\text {Correct }}: \mathrm{t}_{(29)}=0.055, \mathrm{p}=0.956$, mCherry-only: InSeq correct $: \mathrm{t}_{(26)}=-0.457, \mathrm{p}=0.652$, mCherryonly: OutSeq $\left.{ }_{\text {Correct: }} \mathrm{t}_{(26)}=-0.707, \mathrm{p}=0.486\right)$. We followed up this analysis by evaluating the nose-poke distributions in the $\mathrm{hM}_{4} \mathrm{Di}^{+}$group for all InSeq and OutSeq trials following PER infusions (Figures $5 \mathrm{~J}$ and $5 \mathrm{~K}$ ). The proportion of nose pokes in the InSeq distribution was similar between Veh and CNO infusions in the hM4Di+ group (Figure 5J). As shown in Figure 5K, CNO infusion led to a decrease in the proportion of OutSeq nose pokes near the short poke distribution, ( .20-.4 s) and an increase near the 1-s decision threshold, compared with Veh infusion. Thus, the nose-poke differences indicate a decisional shift in the $\mathrm{hM}_{4} \mathrm{Di}^{+}$group following CNO administration. Similar to $\mathrm{mPFC} \rightarrow \mathrm{RE}$, these results illustrate that silencing $\mathrm{mPFC} \rightarrow \mathrm{PER}$ leads to inaccurate decisions rather than a basic deficit in nose-poke and hold behavior, reflecting deficits in sequence memory.

\section{Differential Role of mPFC Top-Down Inputs to RE and PER}

The results demonstrate activity in both $\mathrm{mPFC} \rightarrow \mathrm{RE}$ and $\mathrm{mPFC} \rightarrow$ PER pathways are essential to sequence memory. We next directly compared the effects of silencing $\mathrm{mPFC} \rightarrow \mathrm{RE}$ and $\mathrm{mPFC} \rightarrow \mathrm{PER}$ projections. Overall, the effects of $\mathrm{mPFC} \rightarrow \mathrm{RE}$ and $\mathrm{mPFC} \rightarrow$ PER pathway silencing did not differ significantly from each other across repeated conditions in the $\mathrm{hM} 4 \mathrm{Di}^{+}$group (region: $F_{(1,8)}=1.487, p=0.257$; repeated condition: $\mathrm{F}_{(2,16)}=0.291, \mathrm{p}=0.667$; repeated condition $\times$ region: $\mathrm{F}_{(2,16)}=$ $0.343, p=0.632)$. We then examined whether silencing $\mathrm{mPFC} \rightarrow \mathrm{RE}$ and $\mathrm{mPFC} \rightarrow \mathrm{PER}$ pathways impaired different memory retrieval strategies that support sequence memory. Our approach was based on the conceptual model shown in Figure $6 \mathrm{~A}$. This model illustrates the theoretical performance curves that would be obtained when using working memory and temporal context memory retrieval strategies plotted as a function of sequential lag distances on OutSeq items. With a working memory strategy, repeated items would be easier to detect at shorter lags because these items occurred more recently. Conversely, with a temporal context memory strategy, repeated items would be easier to detect at longer lags because these items are further away in the original sequence, thereby reducing interference. Therefore, we examined the performance of the OutSeq probe trials across lags, focusing on repeated items (also called backward lags) to test the contributions of temporal context memory and working memory. For this analysis, we calculated the percent change in performance (CNO-Veh) for each rat on each lag, as SMI could not be calculated because we were only analyzing OutSeq trials.

We first looked at items that were repeated in a sequence to see whether silencing $\mathrm{mPFC} \rightarrow \mathrm{RE}$ and $\mathrm{mPFC} \rightarrow \mathrm{PER}$ projections differentially affected impairment patterns. $\mathrm{mPFC} \rightarrow \mathrm{RE}$ silencing resulted in significant differences from no change in performance $(0 \%)$ on all backward lags (3-back, $t_{(14)}=-2.388$, $\mathrm{p}=0.016 ; 2$-back, $\mathrm{t}_{(26)}=-4.252, \mathrm{p}=1.21 \times 10^{-4} ; 1$-back, 


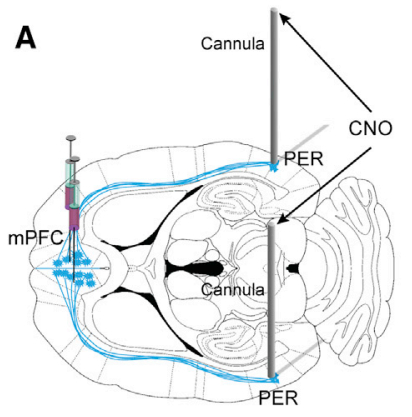

B
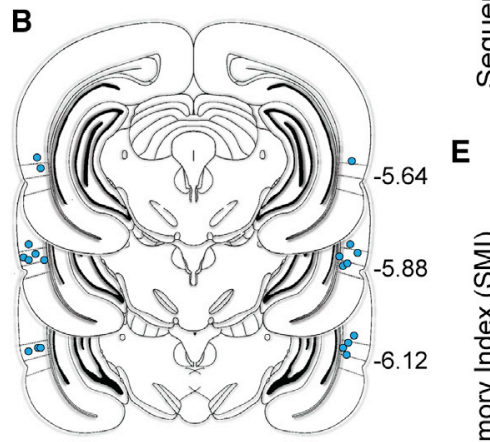

C

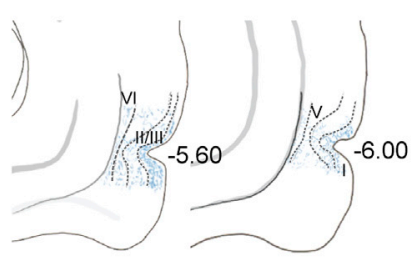

D

\section{E}

ह⿹

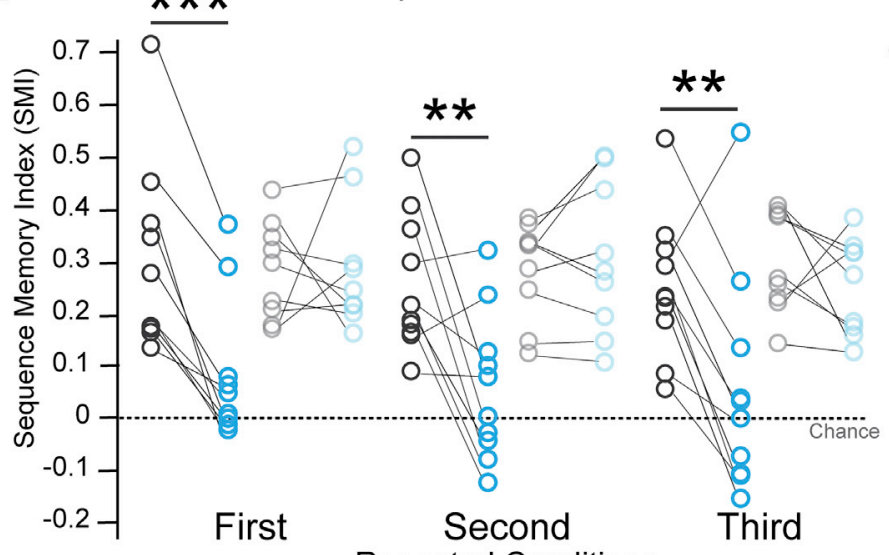

Sequence Memory by Repeated Conditions $\mathbf{F}$

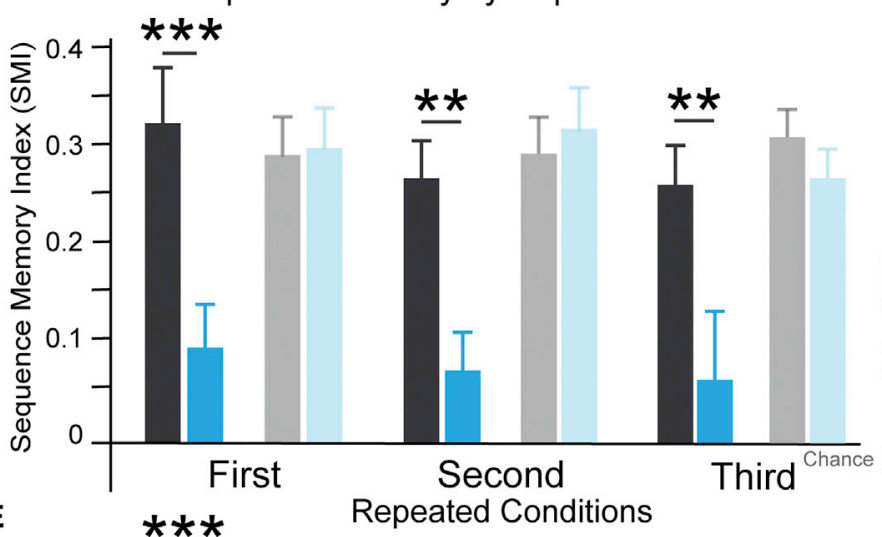

Repeated Conditions
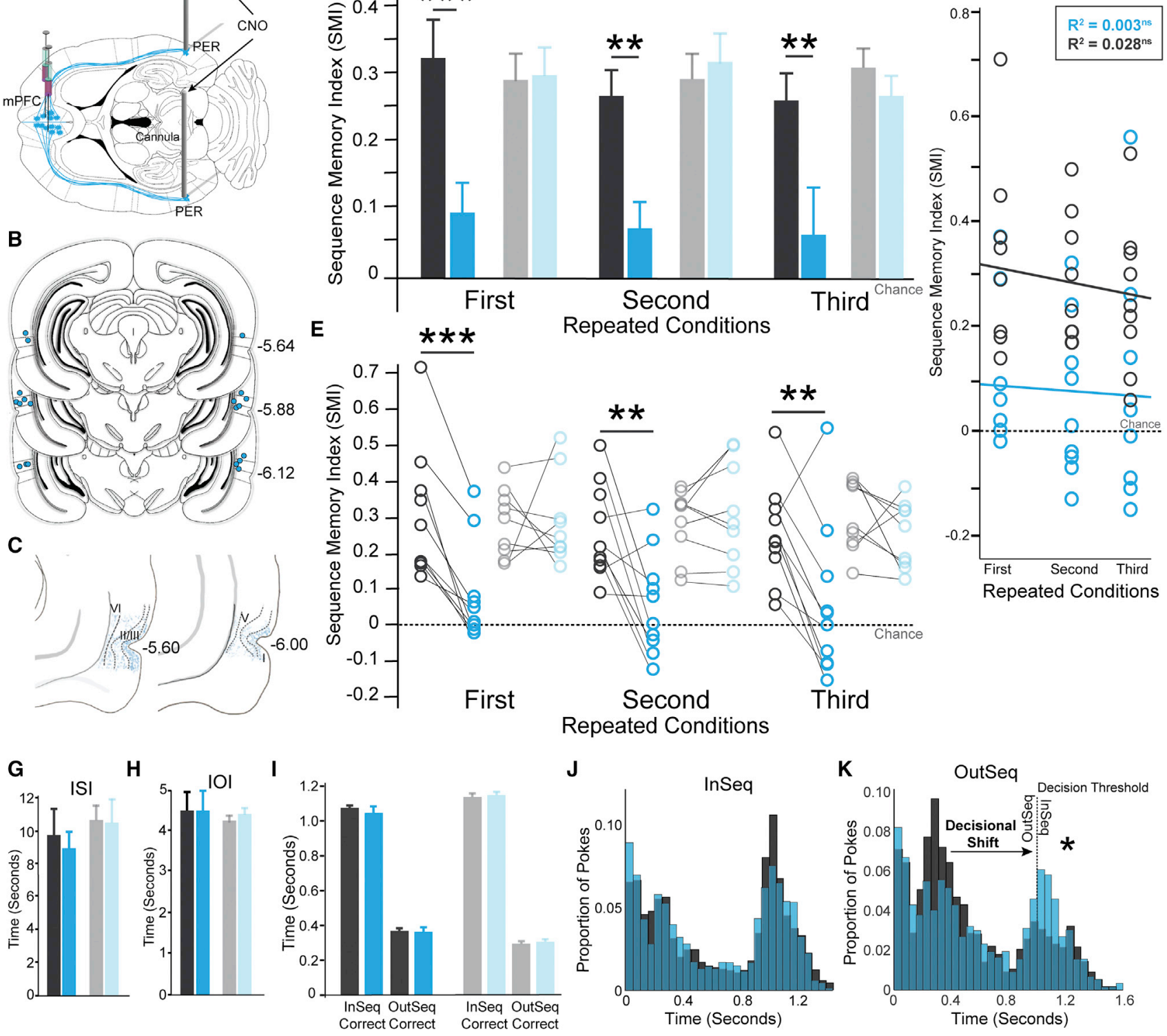

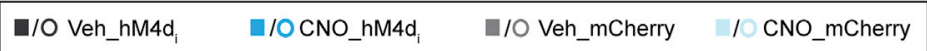

Figure 5. Synaptic Silencing of $\mathrm{mPFC} \rightarrow$ PER Pathway Abolished Sequence Memory (A) Guide cannulas into the PER.

(B) Locations of PER infusion cannulas in all rats $(n=10)$.

(C) Schematic representation of mPFC hM4Di fiber density in PER (restricted to region of interest).

(D) In the hM4Di+ group, SMI significantly differed between the Veh and CNO conditions for all three repeated conditions. No differences between Veh and CNO were detected in the mCherry-only group.

(E) Individual rat performance in each repeated condition for both groups.

(F) In the hM4Di+ group, there was no significant relationship between repeated conditions and infusions.

(G) ISI was not significantly different between PER Veh and CNO conditions for either group.

(H) IOI was not significantly different between PER Veh and CNO infusions for either group.

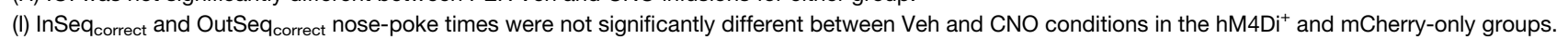
(J) $\mathrm{hM} 4 \mathrm{Di}^{+}$group nose-poke times show no obvious shifts in nose-poking behavior.

(K) hM4Di+ group nose-poke times show a decisional shift (indicated by a star) where the rats incorrectly identified OutSeq odors as InSeq.

All data are represented as mean \pm SEM. ${ }^{*} p<0.05 ;{ }^{\star \star} p<0.01$; ${ }^{\star \star \star} p<0.001$; ns, not significant. 


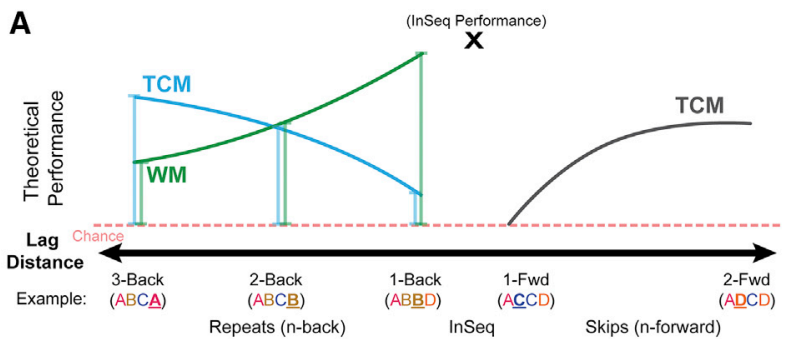

B

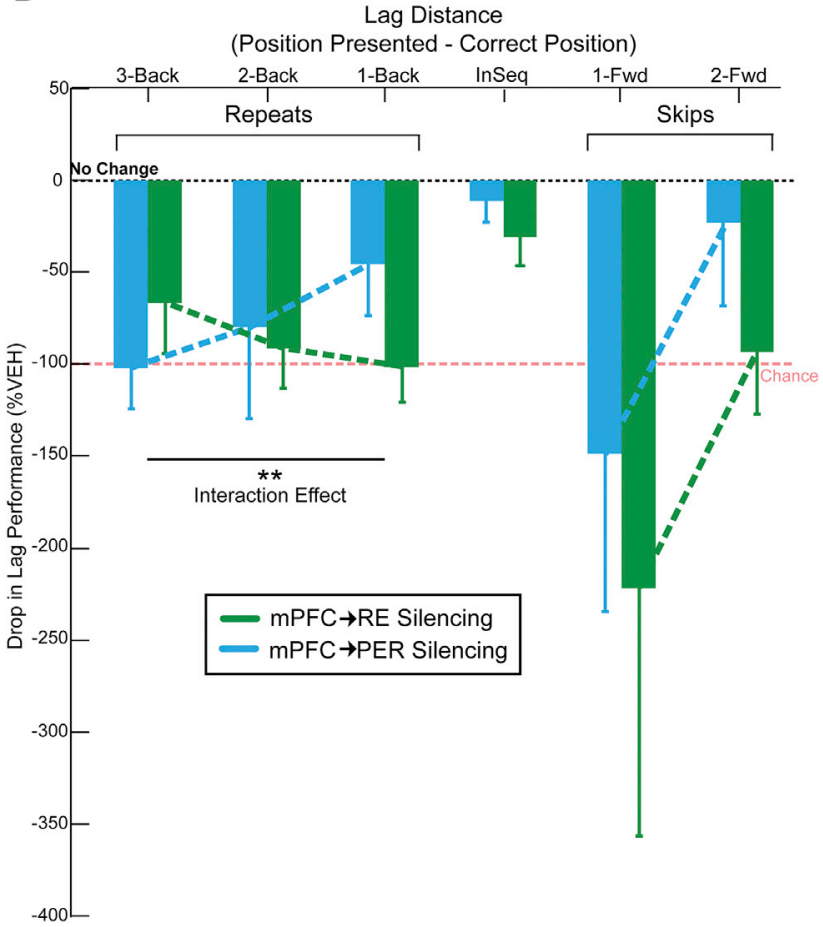

Figure 6. The $\mathrm{mPFC} \rightarrow \mathrm{RE}$ Pathway Supports a Working Memory Retrieval Strategy, while $\mathrm{mPFC} \rightarrow$ PER Pathway Supports a Temporal Context Memory Retrieval Strategy

(A) If rats were using a working memory strategy, then repeated items would be easiest at short lags. By contrast, if rats were using a temporal context memory strategy, then repeated items would be the easiest to detect with longer lags. (B) $\mathrm{mPFC} \rightarrow \mathrm{RE}$ silencing impaired performance most on shorter distances, consistent with a loss of working memory, while mPFC $\rightarrow$ PER silencing impaired performance most at longer distance, consistent with a loss of temporal context memory.

All data are represented as mean \pm SEM. ${ }^{*} p<0.01$.

$\left.t_{(29)}=-5.274, p=6.00 \times 10^{-6}\right)$. Additionally, mPFC $\rightarrow$ PER silencing resulted in a significant difference on the 3-back lag, and showed trends toward significant differences from no change in performance on the 2-back and 1-back lags (3-back, $\mathrm{t}_{(21)}=-4.579, \mathrm{p}=8.15 \times 10^{-5}$; 2-back, $\mathrm{t}_{(27)}=-1.609$, $\mathrm{p}=0.059 ; 1$-back, $\left.\mathrm{t}_{(28)}=-1.639, \mathrm{p}=0.056\right)$. These results indicate repeated items were affected by silencing both $\mathrm{mPFC} \rightarrow \mathrm{RE}$ and $\mathrm{mPFC} \rightarrow$ PER pathways. An important question is whether the performance patterns differed across lags when directly comparing the effects of $\mathrm{mPFC} \rightarrow \mathrm{RE}$ and $\mathrm{mPFC} \rightarrow$ PER pathway silencing. A repeated-measures ANOVA revealed a significant interaction between $\mathrm{mPFC} \rightarrow \mathrm{RE}$ silencing and $\mathrm{mPFC} \rightarrow \mathrm{PER}$ silencing (region $\times$ backward: $F_{(2,24)}=5.395, p=0.012$ ), with a large effect size $\left(y_{p}{ }^{2}=0.310\right.$; Cohen, 1973), but no significant main effects of region or backward lag distance (regions: $F_{(1,12)}=0.721, p=0.413$; backward: $\left.F_{(2,24)}=0.202, p=0.732\right)$. In the $\mathrm{hM}_{4} \mathrm{Di}^{+}$group, CNO infusions in RE had the largest performance-impairing effect on 1-back, then 2-back, and the smallest performance-impairing effect on 3-back lags (Figure 6B). In the $\mathrm{hM}_{4 \mathrm{DI}}{ }^{+}$group, CNO infusions into the PER led to the opposite pattern, with the largest performance drop on 3-back, then 2-back, and the smallest performance drop on 1-back lags (Figure $6 \mathrm{~B})$. These impairment patterns match the expected performance decrements (drop lines in Figure $6 A$ ) from a selective loss of working memory following $\mathrm{mPFC} \rightarrow \mathrm{RE}$ silencing, and a selective loss in temporal context memory following $\mathrm{mPFC} \rightarrow \mathrm{PER}$ silencing.

We then evaluated performance during InSeq trials (lag $=0$ ) against no change in performance $(0 \%)$ following $\mathrm{mPFC} \rightarrow \mathrm{RE}$ and $\mathrm{mPFC} \rightarrow$ PER silencing and found no significant differences $\left(\right.$ InSeq $_{\mathrm{RE}}: \mathrm{t}_{(29)}=-1.189, \mathrm{p}=0.122 ;$ InSeq ${ }_{\mathrm{PER}}: \mathrm{t}_{(28)}=-1.004$, $p=0.162$ ). The finding suggests InSeq trials were not affected by silencing $\mathrm{mPFC} \rightarrow \mathrm{RE}$ and $\mathrm{mPFC} \rightarrow$ PER pathways. We found no significant differences comparing $\mathrm{mPFC} \rightarrow \mathrm{RE}$ and $\mathrm{mPFC} \rightarrow$ PER on InSeq performance levels using a paired-samples $t$ test $\left(\mathrm{t}_{(28)}=-0.982, \mathrm{p}=0.334\right)$.

Finally, we examined whether performance on items that skipped ahead in the sequence (forward lags) were affected by $\mathrm{mPFC} \rightarrow \mathrm{RE}$ and $\mathrm{mPFC} \rightarrow \mathrm{PER}$ pathway silencing. $\mathrm{mPFC} \rightarrow \mathrm{RE}$ pathway silencing tended to have a significant effect on 1-forward $\left(\mathrm{t}_{(29)}=-1.644, \mathrm{p}=0.055\right)$ and 2-forward $\left(\mathrm{t}_{(29)}=-2.725\right.$, $p=0.006$ ) lags. $m P F C \rightarrow P E R$ silencing had a significant effect on the 1-forward lag $\left(\mathrm{t}_{(28)}=1.732, \mathrm{p}=0.047\right)$, and no significant effect on the 2-forward lag $\left(t_{(27)}=-0.524, p=0.303\right)$. Overall, this finding suggests performance levels on items that skipped ahead in the sequence were affected by silencing $\mathrm{mPFC} \rightarrow \mathrm{RE}$ and $\mathrm{mPFC} \rightarrow$ PER pathways. Direct comparison of the effects of silencing $\mathrm{mPFC} \rightarrow \mathrm{RE}$ and $\mathrm{mPFC} \rightarrow \mathrm{PER}$ pathways was performed using repeated-measures ANOVA and revealed no significant interactions in the forward lag direction (region $\times$ forward: $\left.F_{(1,27)}=-0.012, p=0.915\right)$.

\section{DISCUSSION}

\section{Summary of Main Findings}

We evaluated the hypothesis that top-down prefrontal projections contribute to sequence memory, and separate projections control the execution of different retrieval mechanisms. We first established that two non-overlapping populations of mPFC neurons project to RE and PER. We then demonstrated suppressing mPFC activity impairs sequence memory, indicating mPFC is critical to sequence memory, consistent with other reports (e.g., Hannesson et al., 2004; Devito and Eichenbaum, 2011). This finding alone, however, does not address the role of mPFC as top-down control of sequence memory. Therefore, we directly manipulated mPFC circuitry using an hM4Di synaptic-silencing approach. Suppressing activity in $\mathrm{mPFC} \rightarrow \mathrm{RE}$ or $\mathrm{mPFC} \rightarrow$ PER pathway effectively abolished sequence memory. These results unambiguously demonstrate top-down mPFC 
projections are essential to sequence memory. Next, we performed a behavioral lag analysis to determine the differential roles of $\mathrm{mPFC} \rightarrow \mathrm{RE}$ and $\mathrm{mPFC} \rightarrow$ PER pathways. Silencing $\mathrm{mPFC} \rightarrow \mathrm{RE}$ pathway disrupted backward lags with a pattern resembling a loss of working memory, whereas silencing $\mathrm{mPFC} \rightarrow$ PER pathway disrupted backward lags with a pattern resembling a loss of temporal context memory. Theoretically, working memory and temporal context memory (i.e., graded retrieval strength based on temporal proximity) differentially contribute to retrieval performance when items are repeated. On shorter lags (e.g., ABCC, lag = -1 ), working memory strategies lead to better performance and suppressing $m P F C \rightarrow R E$ activity impairs performance. At longer lags (e.g., ABCA, lag $=-3$ ), temporal context memory strategies lead to better performance and suppressing $\mathrm{mPFC} \rightarrow$ PER activity impairs performance. This strong pathway-specific interaction effect demonstrates, for the first time, top-down mPFC projections control sequence memory, and suggests $R E$ and $P E R$ pathways regulate ongoing retrieval strategies.

\section{Testing mPFC Projections with DREADDs in Sequence Memory}

To investigate the top-down role of MPFC in sequence memory, we suppressed synaptic activity in specific mPFC projections (Sesack et al., 1989; Chiba et al., 2001; Vertes, 2002; Hoover and Vertes, 2007) using Gi-coupled (hM4Di) DREADDs (Mahler et al., 2014; Roth, 2016) combined with chronic cannulas targeting $R E$ and PER in rats. hM4Di activation in presynaptic terminals reduces transmission and effectively silences the pathway (Stachniak et al., 2014; Lichtenberg et al., 2017). Here, we focused on terminal fields in RE (Sesack et al., 1989; Chiba et al., 2001; Vertes, 2002; Hoover and Vertes, 2007; Vertes et al., 2015) and PER (Furtak et al., 2007) because both regions support bidirectional communication between the HC and mPFC (e.g., Allen and Fortin, 2013; Vertes et al., 2015; Eichenbaum, 2017b). To confirm our manipulations localized to these pathways, we carefully mapped mPFC hM4Di expression areas, visualized $\mathrm{mPFC} \rightarrow \mathrm{RE}$ and $\mathrm{mPFC} \rightarrow$ PER projection terminals via the co-expression of mCherry (enhanced with IHC), and mapped the tip locations of the infusion cannulas within RE and PER.

It is important to control for non-specific CNO or infusion effects when using hM4Di to evaluate brain-behavior relationships (Smith et al., 2016; Gomez et al., 2017). Thus, we used a fully crossed 2 (hM4Di and hM4Di-free) $\times 2$ (CNO and Veh) experimental design. Notably, we only observed effects of any of our manipulations when we activated hM4Di with CNO and tested sequence memory.

We also performed several analyses to test the alternative hypothesis that non-memory-related behavioral effects account for impaired performance in the sequence task. We saw no effects (under any condition) on the time it took rats to run between sequences, on odor sampling, on reward retrieval activity, or on the overall frequency of nose pokes in which the rats held the nosepoke response for $>1 \mathrm{~s}$ or $<1 \mathrm{~s}$ (analyzed by ignoring the sequential status of items; see Results). In fact, detailed analysis of all nose-poke times under $\mathrm{hM}_{4} \mathrm{Di}^{+}$and $\mathrm{CNO}$ conditions revealed a pattern resembling a shift toward inaccurate OutSeq decisions. This was most clearly revealed in the analysis of poke time histograms and the increase in the overall frequency of holds on OutSeq items, without large changes in the distribution variability or peak times. If the rats had shifted to simple reaction time behaviors, we would have expected an increase in premature and variable responses following $\mathrm{mPFC}$ inactivation (Narayanan and Laubach, 2006). Finally, the sequence memory effects were consistent across two different sequences. This is important because it eliminates the possibility rats held a single sequence in working memory and focused on a single strategy throughout the entirety of the session, but instead rats were forced to repeatedly retrieve sequences from long-term memory stores.

An important observation we made following repeated i.p. injections was a loss in the behavioral effect on sequence memory, whereas with direct infusions (which were performed after the i.p. injections) we did not see this loss. Varela et al. (2016) saw a similar effect observing a weak, but significant, CNO-dependent decrease in freezing levels after multiple injections. There are several possible reasons for the decreased efficacy, including changes in the $\mathrm{CNO} /$ clozapine metabolism, receptor desensitization, and/or compensatory plasticity across the brain. The fact that direct infusions never lost efficacy might argue in favor of a systemic metabolic effect. However, experiments that directly measure CNO and clozapine levels in blood and cerebral spinal fluid following repeated i.p. injections would be needed to test this hypothesis.

\section{mPFC Pathways to RE and PER Control Retrieval} Strategies in Sequence Memory

Generally, mPFC is considered to have a major role in controlling memory retrieval strategies (Shimamura, 1995; Dobbins et al., 2002; Euston et al., 2012; Preston and Eichenbaum, 2013; Jadhav et al., 2016; Eichenbaum, 2017b). We tested this in two specific projection pathways during memory for sequences of events. Importantly, the sequence task we used is related to episodic-like memory processing and depends on the use of multiple cognitive strategies for optimal performance (Allen et al., 2014, 2015).

If rats were using a working memory strategy, then repeated items would be easiest to detect at short lags because those items occurred more recently. On the other hand, if rats were using a temporal context memory strategy (i.e., graded retrieval probabilities determined by the temporal proximity of items in the sequence), then repeated items would be the easiest to detect with longer lags because of reduced interference. Our use of the term temporal context memory refers to the retrieval gradients that are observed in human studies of list learning (e.g., Kragel et al., 2015; Howard and Kahana, 2002). When cued with an item from the list, people will tend to remember the nearby items (short lags), but they are less likely to recall distal items (longer lags). Because verbal recall cannot be performed in animals, the odor sequence task models list learning in rodents using a nonverbal response to probe memory. Temporal context memory is tested with a lag analysis where we expect nearby items to interfere with OutSeq decisions, but not distal items. In the task, working memory is simultaneously tested on reverse lags because delay-match-to-sample strategies can contribute to performance on these trials for nearby 
items, but less so on distal items (the opposite pattern expected in temporal context memory). Thus, the sequence task places pressure on the ability of rats to regulate retrieval strategies at different lag distances for optimal performance by emphasizing working memory on shorter lags and temporal context memory on longer lags. Here, suppressing $\mathrm{mPFC} \rightarrow \mathrm{RE}$ activity had the largest effect on lags $=-1$ and the smallest effect on lags = -3 . This pattern resembles a reduction in a working memory retrieval strategy, is consistent with several studies demonstrating a role for RE in working memory tasks including spatial working memory (Cassel et al., 2013; Griffin, 2015; Layfield et al., 2015; Vertes et al., 2015; Hallock et al., 2016; Maisson et al., 2018; Viena et al., 2018) and visual-tactile working memory (Hallock et al., 2013), and is similar to the role of RE in fear memory retrieval (Ramanathan et al., 2018).

In contrast, we found that suppressing $\mathrm{mPFC} \rightarrow \mathrm{PER}$ had the largest effect on lags $=-3$ and the smallest effect on lags = -1 . This opposite pattern resembles a reduction in a temporal context memory retrieval strategy. Although temporal context memory is primarily attributed to the $\mathrm{HC}$ (Howard and Kahana, 2002; Hsieh et al., 2014; Roberts et al., 2014; Kragel et al., 2015), PER may also be involved in temporal context memory. For example, PER is essential for trace conditioning (KholodarSmith et al., 2008b; Bang and Brown, 2009) and for unitizing representations of discontinuous events (Kholodar-Smith et al., 2008a; Suter et al., 2013). Alternatively, the present results could reflect a role of PER in disambiguating overlapping object features (e.g., Murray and Bussey, 1999), as sequences contain mostly the same elements (e.g., ABCA versus $A B C B$ ), but this idea would not account for the deficit gradients observed as a function of lag distance. Furthermore, PFC-PER disconnection lesions impair rule shifting in a conditional object-place association task, consistent with a pathway-specific role in behavioral flexibility (Hernandez et al., 2017). Generally, the current results demonstrate, for the first time, RE and PER have specific roles in memory for sequences of events, rats use multiple retrieval strategies during sequence memory, and these strategies can be differentially controlled by reducing $\mathrm{mPFC} \rightarrow \mathrm{RE}$ and $\mathrm{mPFC} \rightarrow$ PER pathway activity.

Interestingly, rats showed a similar level of performance deficits on items that skipped ahead in the sequence (e.g., ABD) following suppression of both $\mathrm{mPFC} \rightarrow \mathrm{RE}$ and $\mathrm{mPFC} \rightarrow \mathrm{PER}$. Theoretically, only temporal context memory or ordinal representational strategies (which were not tested here; see Orlov et al., 2000; Reeders et al., 2018) can support accurate performance when predicting upcoming items. Thus, these results indicate both $\mathrm{mPFC} \rightarrow \mathrm{RE}$ and $\mathrm{mPFC} \rightarrow \mathrm{PER}$ are critical for making predictions of upcoming items. The exact nature of each pathway's contribution to memory, however, should be further explored in experiments focused on items skipping ahead farther in sequences, by manipulating positional strategies, and in tasks focused on elapsed time memory across multiple delay periods (e.g., Jacobs et al., 2013; MacDonald et al., 2013).

\section{Conclusions}

We present evidence that top-down mPFC pathways targeting $\mathrm{RE}$ and PER differentially control the retrieval strategy used to support sequence memory. Generally, the ability to shift retrieval strategies is important for situation-specific memory access and optimal memory-guided behavior. Importantly, RE and PER pathways endow mPFC with the ability to exert top-down control over episodic memory. Future studies should investigate whether these pathways are vulnerable in disorders that affect the temporal organization of memory, such as schizophrenia and Alzheimer disease.

\section{STAR $\star$ METHODS}

Detailed methods are provided in the online version of this paper and include the following:

- KEY RESOURCES TABLE

- LEAD CONTACT AND MATERIALS AVAILABILITY

- EXPERIMENTAL MODEL AND SUBJECT DETAILS

- METHOD DETAILS

O Sequence memory task

○ Task apparatus

O Sequence memory task training

O Cannula system

O AAV9 microsyringe infusions and cannula placement

O Suppressing mPFC neurons and projections

Dual retrograde tracing

O Histology

- QUANTIFICATION AND STATISTICAL ANALYSIS

$O$ Quantification of mPFC cells and fiber density in RE and PER

Statistics

\section{SUPPLEMENTAL INFORMATION}

Supplemental Information can be found online at https://doi.org/10.1016/j. celrep.2019.06.053.

\section{ACKNOWLEDGMENTS}

This work was supported by NIH grant R01 MH113626. Thanks to members of the Allen laboratory, including Dr. L.M. Allen, Dr. T. Viena, J. Perez, R. Viena, and C. Pavon, who helped with the data collection and A. Rojas for her assistance with schematic renderings. We thank Dr. A.T. Mattfeld and A.G. Hamm for useful feedback on the manuscript. We would like to thank the Animal Care Facility and Dr. H. Vinerean.

\section{AUTHOR CONTRIBUTIONS}

Conceptualization, T.A.A. and M.J.; Methodology, T.A.A., S.V.M., and M.J.; Investigation, M.J., M.S., and S.B.L.; Writing - Original Draft, M.J., S.B.L., and T.A.A.; Writing - Review and Editing, T.A.A., S.B.L., R.P.V., S.V.M., M.S., and M.J.; Funding Acquisition, T.A.A. and R.P.V.; Resources, T.A.A. and R.P.V.; Supervision, T.A.A.

\section{DECLARATION OF INTERESTS}

The authors declare no competing interests.

Received: February 8, 2019

Revised: May 19, 2019

Accepted: June 14, 2019

Published: July 16, 2019 


\section{REFERENCES}

Aggleton, J.P., Albasser, M.M., Aggleton, D.J., Poirier, G.L., and Pearce, J.M. (2010). Lesions of the rat perirhinal cortex spare the acquisition of a complex configural visual discrimination yet impair object recognition. Behav. Neurosci. 124, 55-68.

Agster, K.L., Fortin, N.J., and Eichenbaum, H. (2002). The hippocampus and disambiguation of overlapping sequences. J. Neurosci. 22, 5760-5768.

Allen, T.A., and Fortin, N.J. (2013). The evolution of episodic memory. Proc. Natl. Acad. Sci. USA 110 (Supp/ 2), 10379-10386.

Allen, T.A., Furtak, S.C., and Brown, T.H. (2007). Single-unit responses to 22 $\mathrm{kHz}$ ultrasonic vocalizations in rat perirhinal cortex. Behav. Brain Res. 182, 327-336.

Allen, T.A., Morris, A.M., Mattfeld, A.T., Stark, C.E., and Fortin, N.J. (2014). A sequence of events model of episodic memory shows parallels in rats and humans. Hippocampus 24, 1178-1188.

Allen, T.A., Morris, A.M., Stark, S.M., Fortin, N.J., and Stark, C.E. (2015). Memory for sequences of events impaired in typical aging. Learn. Mem. 22, 138-148.

Allen, T.A., Salz, D.M., McKenzie, S., and Fortin, N.J. (2016). Nonspatial sequence coding in CA1 neurons. J. Neurosci. 36, 1547-1563.

Bang, S.J., and Brown, T.H. (2009). Muscarinic receptors in perirhinal cortex control trace conditioning. J. Neurosci. 29, 4346-4350.

Barker, G.R., and Warburton, E.C. (2011). Evaluating the neural basis of temporal order memory for visual stimuli in the rat. Eur. J. Neurosci. 33, 705-716.

Barker, G.R., Bird, F., Alexander, V., and Warburton, E.C. (2007). Recognition memory for objects, place, and temporal order: a disconnection analysis of the role of the medial prefrontal cortex and perirhinal cortex. J. Neurosci. 27, 29482957.

Blumenfeld, R.S., Parks, C.M., Yonelinas, A.P., and Ranganath, C. (2011). Putting the pieces together: the role of dorsolateral prefrontal cortex in relational memory encoding. J. Cogn. Neurosci. 23, 257-265.

Bussey, T.J., Saksida, L.M., and Murray, E.A. (2002). Perirhinal cortex resolves feature ambiguity in complex visual discriminations. Eur. J. Neurosci. 15, 365-374.

Bussey, T.J., Saksida, L.M., and Murray, E.A. (2005). The perceptual-mnemonic/feature conjunction model of perirhinal cortex function. Q. J. Exp. Psychol. B 58, 269-282.

Cassel, J.C., Pereira de Vasconcelos, A., Loureiro, M., Cholvin, T., DalrympleAlford, J.C., and Vertes, R.P. (2013). The reuniens and rhomboid nuclei: neuroanatomy, electrophysiological characteristics and behavioral implications. Prog. Neurobiol. 111, 34-52.

Castle, M.J., Perlson, E., Holzbaur, E.L., and Wolfe, J.H. (2014). Long-distance axonal transport of AAV9 is driven by dynein and kinesin-2 and is trafficked in a highly motile Rab7-positive compartment. Mol. Ther. 22, 554-566.

Chen, Q., Garcea, F.E., and Mahon, B.Z. (2016). The representation of objectdirected action and function knowledge in the human brain. Cereb. Cortex 26, 1609-1618.

Chiba, T., Kayahara, T., and Nakano, K. (2001). Efferent projections of infralimbic and prelimbic areas of the medial prefrontal cortex in the Japanese monkey, Macaca fuscata. Brain Res. 888, 83-101.

Clayton, N.S., and Dickinson, A. (1998). Episodic-like memory during cache recovery by scrub jays. Nature 395, 272-274.

Cohen, J. (1973). Eta-squared and partial eta-squared in fixed factor ANOVA designs. Educ. Psychol. Meas. 33, 107-112.

Davoodi, F.G., Motamedi, F., Naghdi, N., and Akbari, E. (2009). Effect of reversible inactivation of the reuniens nucleus on spatial learning and memory in rats using Morris water maze task. Behav. Brain Res. 198, 130-135.

Devito, L.M., and Eichenbaum, H. (2011). Memory for the order of events in specific sequences: contributions of the hippocampus and medial prefrontal cortex. J. Neurosci. 31, 3169-3175.
DiBenedictis, B.T., Olugbemi, A.O., Baum, M.J., and Cherry, J.A. (2015) DREADD-induced silencing of the medial olfactory tubercle disrupts the preference of female mice for opposite-sex chemosignals. eNeuro 2, 1-16.

Dobbins, I.G., Foley, H., Schacter, D.L., and Wagner, A.D. (2002). Executive control during episodic retrieval: multiple prefrontal processes subserve source memory. Neuron 35, 989-996.

Dolleman-van der Weel, M.J., Morris, R.G., and Witter, M.P. (2009). Neurotoxic lesions of the thalamic reuniens or mediodorsal nucleus in rats affect non-mnemonic aspects of watermaze learning. Brain Struct. Funct. 213, 329-342.

Eichenbaum, H. (2004). Hippocampus: cognitive processes and neural representations that underlie declarative memory. Neuron 44, 109-120.

Eichenbaum, H. (2017a). On the integration of space, time, and memory. Neuron 95, 1007-1018.

Eichenbaum, H. (2017b). Prefrontal-hippocampal interactions in episodic memory. Nat. Rev. Neurosci. 18, 547-558.

Euston, D.R., Tatsuno, M., and McNaughton, B.L. (2007). Fast-forward playback of recent memory sequences in prefrontal cortex during sleep. Science $318,1147-1150$

Euston, D.R., Gruber, A.J., and McNaughton, B.L. (2012). The role of medial prefrontal cortex in memory and decision making. Neuron 76, 1057-1070.

Feinberg, L.M., Allen, T.A., Ly, D., and Fortin, N.J. (2012). Recognition memory for social and non-social odors: differential effects of neurotoxic lesions to the hippocampus and perirhinal cortex. Neurobiol. Learn. Mem. 97, 7-16.

Ferbinteanu, J., Kennedy, P.J., and Shapiro, M.L. (2006). Episodic memoryfrom brain to mind. Hippocampus 16, 691-703.

Fortin, N.J., Agster, K.L., and Eichenbaum, H.B. (2002). Critical role of the hippocampus in memory for sequences of events. Nat. Neurosci. 5, 458-462.

Furtak, S.C., Wei, S.M., Agster, K.L., and Burwell, R.D. (2007). Functional neuroanatomy of the parahippocampal region in the rat: the perirhinal and postrhinal cortices. Hippocampus 17, 709-722.

Gomez, J.L., Bonaventura, J., Lesniak, W., Mathews, W.B., Sysa-Shah, P., Rodriguez, L.A., Ellis, R.J., Richie, C.T., Harvey, B.K., Dannals, R.F., et al. (2017). Chemogenetics revealed: DREADD occupancy and activation via converted clozapine. Science 357, 503-507.

Griffin, A.L. (2015). Role of the thalamic nucleus reuniens in mediating interactions between the hippocampus and medial prefrontal cortex during spatia working memory. Front. Syst. Neurosci. 9, 29.

Hales, J.B., Israel, S.L., Swann, N.C., and Brewer, J.B. (2009). Dissociation of frontal and medial temporal lobe activity in maintenance and binding of sequentially presented paired associates. J. Cogn. Neurosci. 21, 1244-1254.

Hallock, H.L., Wang, A., Shaw, C.L., and Griffin, A.L. (2013). Transient inactivation of the thalamic nucleus reuniens and rhomboid nucleus produces deficits of a working-memory dependent tactile-visual conditional discrimination task. Behav. Neurosci. 127, 860-866.

Hallock, H.L., Wang, A., and Griffin, A.L. (2016). Ventral midline thalamus is critical for hippocampal-prefrontal synchrony and spatial working memory. J. Neurosci. 36, 8372-8389.

Hannesson, D.K., Howland, J.G., and Phillips, A.G. (2004). Interaction between perirhinal and medial prefrontal cortex is required for temporal order but not recognition memory for objects in rats. J. Neurosci. 24, 4596-4604.

Henson, R.N. (2001). Short-term memory for serial order. Psychologist 14 , $70-73$.

Hernandez, A.R., Reasor, J.E., Truckenbrod, L.M., Lubke, K.N., Johnson, S.A., Bizon, J.L., Maurer, A.P., and Burke, S.N. (2017). Medial prefrontal-perirhinal cortical communication is necessary for flexible response selection. Neurobiol. Learn. Mem. 137, 36-47.

Hoover, W.B., and Vertes, R.P. (2007). Anatomical analysis of afferent projections to the medial prefrontal cortex in the rat. Brain Struct. Funct. 212, 149-179.

Hoover, W.B., and Vertes, R.P. (2012). Collateral projections from nucleus reuniens of thalamus to hippocampus and medial prefrontal cortex in the rat: a 
single and double retrograde fluorescent labeling study. Brain Struct. Funct. 217, 191-209.

Howard, M.W., and Kahana, M.J. (2002). When does semantic similarity help episodic retrieval? J. Mem. Lang. 46, 85-98.

Hsieh, L.T., and Ranganath, C. (2015). Cortical and subcortical contributions to sequence retrieval: schematic coding of temporal context in the neocortical recollection network. Neuroimage 121, 78-90.

Hsieh, L.T., Gruber, M.J., Jenkins, L.J., and Ranganath, C. (2014). Hippocampal activity patterns carry information about objects in temporal context. Neuron 81, 1165-1178.

Hwang, E., Willis, B.S., and Burwell, R.D. (2018). Prefrontal connections of the perirhinal and postrhinal cortices in the rat. Behav. Brain Res. 354, 8-21.

Ito, H.T., Zhang, S.J., Witter, M.P., Moser, E.I., and Moser, M.B. (2015). A prefrontal-thalamo-hippocampal circuit for goal-directed spatial navigation. Nature 522, 50-55.

Jacobs, N.S., Allen, T.A., Nguyen, N., and Fortin, N.J. (2013). Critical role of the hippocampus in memory for elapsed time. J. Neurosci. 33, 13888-13893.

Jadhav, S.P., Rothschild, G., Roumis, D.K., and Frank, L.M. (2016). Coordinated excitation and inhibition of prefrontal ensembles during awake hippocampal sharp-wave ripple events. Neuron 90, 113-127.

Kesner, R.P., Gilbert, P.E., and Barua, L.A. (2002). The role of the hippocampus in memory for the temporal order of a sequence of odors. Behav. Neurosci. 116, 286-290.

Kholodar-Smith, D.B., Allen, T.A., and Brown, T.H. (2008a). Fear conditioning to discontinuous auditory cues requires perirhinal cortical function. Behav. Neurosci. 122, 1178-1185.

Kholodar-Smith, D.B., Boguszewski, P., and Brown, T.H. (2008b). Auditory trace fear conditioning requires perirhinal cortex. Neurobiol. Learn. Mem. 90, 537-543.

Knierim, J.J. (2015). The hippocampus. Curr. Biol. 25, R1116-R1121.

Knierim, J.J., Lee, I., and Hargreaves, E.L. (2006). Hippocampal place cells: parallel input streams, subregional processing, and implications for episodic memory. Hippocampus 16, 755-764.

Kragel, J.E., Morton, N.W., and Polyn, S.M. (2015). Neural activity in the medial temporal lobe reveals the fidelity of mental time travel. J. Neurosci. 35, 29142926.

Layfield, D.M., Patel, M., Hallock, H., and Griffin, A.L. (2015). Inactivation of the nucleus reuniens/rhomboid causes a delay-dependent impairment of spatial working memory. Neurobiol. Learn. Mem. 125, 163-167.

Lichtenberg, N.T., Pennington, Z.T., Holley, S.M., Greenfield, V.Y., Cepeda, C., Levine, M.S., and Wassum, K.M. (2017). Basolateral amygdala to orbitofrontal cortex projections enable cue-triggered reward expectations. J. Neurosci. 37, 8374-8384.

MacDonald, C.J., Carrow, S., Place, R., and Eichenbaum, H. (2013). Distinct hippocampal time cell sequences represent odor memories in immobilized rats. J. Neurosci. 33, 14607-14616.

Mahler, S.V., Vazey, E.M., Beckley, J.T., Keistler, C.R., McGlinchey, E.M., Kaufling, J., Wilson, S.P., Deisseroth, K., Woodward, J.J., and Aston-Jones, G. (2014). Designer receptors show role for ventral pallidum input to ventral tegmental area in cocaine seeking. Nat. Neurosci. 17, 577-585.

Maisson, D.J.N., Gemzik, Z.M., and Griffin, A.L. (2018). Optogenetic suppression of the nucleus reuniens selectively impairs encoding during spatial working memory. Neurobiol. Learn. Mem. 155, 78-85.

Mathiasen, M.L., Amin, E., Nelson, A.J.D., Dillingham, C.M., O'Mara, S.M., and Aggleton, J.P. (2019). Separate cortical and hippocampal cell populations target the rat nucleus reuniens and mammillary bodies. Eur. J. Neurosci. 49, 1649-1672.

McKenna, J.T., and Vertes, R.P. (2004). Afferent projections to nucleus reuniens of the thalamus. J. Comp. Neurol. 480, 115-142.

Murray, E.A., and Bussey, T.J. (1999). Perceptual-mnemonic functions of the perirhinal cortex. Trends Cogn. Sci. 3, 142-151.
Murray, E.A., and Richmond, B.J. (2001). Role of perirhinal cortex in object perception, memory, and associations. Curr. Opin. Neurobiol. 11, 188-193.

Murray, E.A., Bussey, T.J., Hampton, R.R., and Saksida, L.M. (2000). The parahippocampal region and object identification. Ann. N Y Acad. Sci. 911, 166-174.

Narayanan, N.S., and Laubach, M. (2006). Top-down control of motor cortex ensembles by dorsomedial prefrontal cortex. Neuron 52, 921-931.

Naya, Y., Chen, H., Yang, C., and Suzuki, W.A. (2017). Contributions of primate prefrontal cortex and medial temporal lobe to temporal-order memory. Proc. Natl. Acad. Sci. USA 114, 13555-13560.

Orlov, T., Yakovlev, V., Hochstein, S., and Zohary, E. (2000). Macaque monkeys categorize images by their ordinal number. Nature 404, 77-80.

Paxinos, G., and Watson, C. (2004). The Rat Brain in Stereotaxic Coordinates, Fifth Edition (Academic Press).

Preston, A.R., and Eichenbaum, H. (2013). Interplay of hippocampus and prefrontal cortex in memory. Curr. Biol. 23, R764-R773.

Ramanathan, K.R., Jin, J., Giustino, T.F., Payne, M.R., and Maren, S. (2018). Prefrontal projections to the thalamic nucleus reuniens mediate fear extinction. Nat. Commun. 9, 4527.

Reeders, P.C., Allen, T.A., and Mattfeld, A.T. (2018). Hippocampus activations reflect temporal contexts while medial prefrontal cortex activations reflect ordinal positions during sequence memory in humans. bioRxiv. https://doi. org/10.1101/501122.

Roberts, J.M., Ly, M., Murray, E., and Yassa, M.A. (2014). Temporal discrimination deficits as a function of lag interference in older adults. Hippocampus 24, 1189-1196.

Roth, B.L. (2016). DREADDs for neuroscientists. Neuron 89, 683-694.

Sesack, S.R., Deutch, A.Y., Roth, R.H., and Bunney, B.S. (1989). Topographical organization of the efferent projections of the medial prefrontal cortex in the rat: an anterograde tract-tracing study with Phaseolus vulgaris leucoagglutinin. J. Comp. Neurol. 290, 213-242.

Shimamura, A.P. (1995). Memory and the prefrontal cortex. Ann. N Y Acad. Sci. 769, 151-159.

Skelin, I., Kilianski, S., and McNaughton, B.L. (2019). Hippocampal coupling with cortical and subcortical structures in the context of memory consolidation. Neurobiol. Learn. Mem. 160, 21-31.

Smith, K.S., Bucci, D.J., Luikart, B.W., and Mahler, S.V. (2016). DREADDS: use and application in behavioral neuroscience. Behav. Neurosci. 130, 137-155.

Sokal, R.R., and Rohlf, F.J. (1995). Biometry: The Principles and Practices of Statistics in Biological Research (W.H. Freeman and Company).

Stachniak, T.J., Ghosh, A., and Sternson, S.M. (2014). Chemogenetic synaptic silencing of neural circuits localizes a hypothalamus $\rightarrow$ midbrain pathway for feeding behavior. Neuron 82, 797-808.

Suter, E.E., Weiss, C., and Disterhoft, J.F. (2013). Perirhinal and postrhinal, but not lateral entorhinal, cortices are essential for acquisition of trace eyeblink conditioning. Learn. Mem. 20, 80-84.

Tervo, D.G.R., Hwang, B.Y., Viswanathan, S., Gaj, T., Lavzin, M., Ritola, K.D., Lindo, S., Michael, S., Kuleshova, E., Ojala, D., et al. (2016). A designer AAV variant permits efficient retrograde access to projection neurons. Neuron 92 372-382.

Tiganj, Z., Shankar, K.H., and Howard, M.W. (2017). Scale invariant value computation for reinforcement learning in continuous time. In Science of Intelligence: Computational Principles of Natural and Artificial Intelligence, Technical Report SS-17-07 (AAAI Spring Symposium Series), pp. 637-642.

Tiganj, Z., Cromer, J.A., Roy, J.E., Miller, E.K., and Howard, M.W. (2018). Compressed timeline of recent experience in monkey lateral prefrontal cortex. J. Cogn. Neurosci. 30, 935-950.

Tulving, E. (2002). Episodic memory: from mind to brain. Annu. Rev. Psychol. $53,1-25$.

Uylings, H.B., Groenewegen, H.J., and Kolb, B. (2003). Do rats have a prefrontal cortex? Behav. Brain Res. 146, 3-17. 
Varela, C., Weiss, S., Meyer, R., Halassa, M., Biedenkapp, J., Wilson, M.A., Goosens, K.A., and Bendor, D. (2016). Tracking the time-dependent role of the hippocampus in memory recall using DREADDs. PLoS ONE 11, e0154374.

Vertes, R.P. (2002). Analysis of projections from the medial prefrontal cortex to the thalamus in the rat, with emphasis on nucleus reuniens. J. Comp. Neurol. 442, 163-187.

Vertes, R.P. (2004). Differential projections of the infralimbic and prelimbic cortex in the rat. Synapse 51, 32-58.

Vertes, R.P. (2006). Interactions among the medial prefrontal cortex, hippocampus and midline thalamus in emotional and cognitive processing in the rat. Neuroscience 142, 1-20.
Vertes, R.P., Hoover, W.B., Do Valle, A.C., Sherman, A., and Rodriguez, J.J. (2006). Efferent projections of reuniens and rhomboid nuclei of the thalamus in the rat. J. Comp. Neurol. 499, 768-796.

Vertes, R.P., Hoover, W.B., Szigeti-Buck, K., and Leranth, C. (2007). Nucleus reuniens of the midline thalamus: link between the medial prefrontal cortex and the hippocampus. Brain Res. Bull. 71, 601-609.

Vertes, R.P., Linley, S.B., and Hoover, W.B. (2015). Limbic circuitry of the midline thalamus. Neurosci. Biobehav. Rev. 54, 89-107.

Viena, T.D., Linley, S.B., and Vertes, R.P. (2018). Inactivation of nucleus reuniens impairs spatial working memory and behavioral flexibility in the rat. Hippocampus 28, 297-311.

Xu, W., and Südhof, T.C. (2013). A neural circuit for memory specificity and generalization. Science 339, 1290-1295. 


\section{STAR $\star$ METHODS}

\section{KEY RESOURCES TABLE}

\begin{tabular}{|c|c|c|}
\hline REAGENT or RESOURCE & SOURCE & IDENTIFIER \\
\hline \multicolumn{3}{|l|}{ Antibodies } \\
\hline Rabbit anti-Red Fluorescent Protein & Rockland, Inc & $600-401-379$ \\
\hline Mouse Anti-NeuN Antibody, clone A60 & Millipore Sigma, Inc. & RRID: AB_2149209; MAB377 \\
\hline Anti-GAD67 Antibody, clone 1G10.2 & Milipore Sigma, Inc. & RRID: AB_2278725; MAB5406 \\
\hline $\begin{array}{l}\text { VectaFluor Duet Immunofluorescence Double } \\
\text { Labeling Kit, DyLight } 594 \text { Anti-Rabbit (red)/DyLight } \\
488 \text { Anti-Mouse (green) }\end{array}$ & Vector Laboratories & DK-8828 \\
\hline Biotinylated Goat Anti-Rabbit IgG Antibody & Vector Laboratories & RRID: AB_2313606; BA-1000 \\
\hline $\begin{array}{l}\text { Goat anti-Mouse IgG }(\mathrm{H}+\mathrm{L}) \text { Cross-Adsorbed } \\
\text { Secondary Antibody, DyLight } 405\end{array}$ & Invitrogen & 35500BID \\
\hline VECTASTAIN Elite ABC HRP Kit & Vector Laboratories & PK-6200 \\
\hline VECTASHIELD Antifade Mounting Medium & Vector Laboratories & $\mathrm{H}-1200$ \\
\hline $\begin{array}{l}\text { VECTASHIELD HardSet Antifade Mounting } \\
\text { Medium }\end{array}$ & Vector Laboratories & $\mathrm{H}-1400$ \\
\hline 3,3'-Diaminobenzidine & Sigma-Aldrich & CAS: $91-95-2$ \\
\hline \multicolumn{3}{|l|}{ Bacterial and Virus Strains } \\
\hline CAG.mCherry-2a-hM4Di ${ }_{i}^{\text {nrxn }}$ & AddGene & 52523 \\
\hline AAV9.CAG.mCherry-2a-hM4D ${ }_{i}^{\text {nrxn }}$.WPRE.SV40 & UPenn Vector Core & Custom \\
\hline AAV9.CB7.CI.mCherry.WPRE.rBG & UPenn Vector Core & 105544-AAV9 \\
\hline Alexa Fluor 488 Cholera Toxin Subunit B & Invitrogen & C-22841 \\
\hline Alexa Fluor 594 Cholera Toxin Subunit B & Invitrogen & C-22842 \\
\hline \multicolumn{3}{|l|}{ Chemicals, Peptides, and Recombinant Proteins } \\
\hline 1-Octanol & Sigma-Aldrich & CAS: $111-87-5$ \\
\hline$(-)$ - Limonene & Sigma-Aldrich & CAS: $5989-54-8$ \\
\hline I-Menthone & Sigma-Aldrich & CAS: $14073-97-3$ \\
\hline Isobutyl Alcohol & Sigma-Aldrich & CAS: $78-83-1$ \\
\hline Acetophenone & Sigma-Aldrich & CAS: $98-86-2$ \\
\hline (1S) - (-) - beta-Pinene & Sigma-Aldrich & CAS: 18172-67-3 \\
\hline L (-) - Carvone & Sigma-Aldrich & CAS: $6485-40-1$ \\
\hline 5-Methyl-2-Hexanone & Sigma-Aldrich & CAS: $110-12-3$ \\
\hline Clozapine-N-Oxide & Caymen Chemicals & ab141704 \\
\hline \multicolumn{3}{|l|}{ Experimental Models: Organisms/Strains } \\
\hline Rat: Long Evans & Charles River Laboratories & RRID: RGD_2308852 \\
\hline \multicolumn{3}{|l|}{ Software and Algorithms } \\
\hline MATLAB (Version R2016a) & $\begin{array}{l}\text { https://www.mathworks.com/products/ } \\
\text { matlab.html }\end{array}$ & RRID: SCR_001622 \\
\hline OmniPlex (Version 1.17) & https://plexon.com & RRID:SCR_014803 \\
\hline \multicolumn{3}{|l|}{ CinePlex (Version 3.6.0) } \\
\hline SPSS (Version 20) & https://www.ibm.com/us-en/?ar=1 & RRID:SCR_002865 \\
\hline Excel 2016 & Microsoft Office & \\
\hline Fluoview FV10-ASW Version 04.02.04.05 & http://www.olympus-lifescience.com/en/ & RRID:SCR_014215 \\
\hline FIJI ImageJ 2.0.0v & https://imagej.nih.gov/ij & RRID:SCR_003070 \\
\hline
\end{tabular}




\section{LEAD CONTACT AND MATERIALS AVAILABILITY}

Requests for further information and requests for resources should be directed to and will be fulfilled by the Lead Contact, Timothy A. Allen (tallen@fiu.edu).

\section{EXPERIMENTAL MODEL AND SUBJECT DETAILS}

All experimental procedures using animals were conducted in accordance with the Florida International University Institutional Animal Care and Use Committee (FIU-IACUC). Male Long-Evans rats ( $=34$; Charles River Laboratories; weighing $250-350 \mathrm{~g}$ upon arrival) were used. Rats were individually housed and maintained on a 12-h inverse light/dark cycle (lights off at 10 AM). Rats had ad libitum access to food, but access to water was limited to 2-5 min each day, depending on how much water they received as a reward during behavioral training $(6-9 \mathrm{ml})$. All training and testing sessions were conducted during the dark phase (active period) of the light cycle.

\section{METHOD DETAILS}

\section{Sequence memory task}

The sequence memory task (Allen et al., 2014) involves repeated presentations of odor-sequences and requires a rat to determine whether each item (odor) was presented in-sequence (InSeq; by holding the nose-poke response for $1 \mathrm{~s}$ ) or out-of-sequence (OutSeq; by withdrawing its nose from the port before $1 \mathrm{~s}$ ). Rats were trained on two sequences, each comprising four distinct odors (e.g., Seq1: $A_{1} B_{1} C_{1} D_{1}$, Seq2: $A_{2} B_{2} C_{2} D_{2}$ ). Each sequence was presented at either end of a linear track maze. Odor presentations were initiated by a nose-poke, and each trial was terminated after the rat either held the nose-poke response for $>1 \mathrm{~s}$ (InSeq) or withdrew its nose from the port before $1 \mathrm{~s}$ (OutSeq). There was a $1 \mathrm{~s}$ interval between trials. Water rewards (one packet of aspartame for every $500 \mathrm{~mL}$ of water) were delivered below the odor port after each correct response. Following an incorrect response, a buzzer sound was emitted and the sequence was terminated. Each sequence was presented 50-100 times per session; approximately half the presentations included all items InSeq (ABCD) and half included one item OutSeq (e.g., ABAD, odor A repeated in the $3^{\text {rd }}$ position). Note OutSeq items could be presented in any sequence position except the first position (i.e., sequences always began with an InSeq item). Sequence memory was probed with OutSeq trials (e.g., ABAD; one OutSeq trial randomly presented per sequence) and lag distances were analyzed to reveal the temporal order memory performance.

\section{Task apparatus}

Rats were tested in a noise-attenuated experimental room. The behavioral apparatus comprised a linear track (length, $183 \mathrm{~cm}$; width, $10 \mathrm{~cm}$; height, $43 \mathrm{~cm}$ ) with walls angled outward at $15^{\circ}$ and nose ports at each end through which repeated deliveries of multiple distinct odors could be presented. Photobeam sensors were used to detect nose port entries. Each nose port was connected to an odor delivery system (Med Associates). Odor deliveries were initiated by a nose-poke entry and terminated either when the rat withdrew before $1 \mathrm{~s}$, or after $1 \mathrm{~s}$ had elapsed. Water ports were positioned under each nose port for reward delivery. Timing boards (Plexon) and digital input/output devices (National Instruments) were used to measure all event times and control the hardware. All aspects of the task were automated using custom MATLAB scripts (MathWorks R2016a). A 256-channel Omniplex D with video tracking and Cineplex behavior software (Plexon) were used to interface with the hardware in real time and record behavioral data. Odors were organic odorants contained in glass jars (A1: 1-octanol, CAS: 111-87-5; B1: (-) - limonene, CAS: 5989-54-8; C1: I-menthone, CAS: 14073-97-3; D1: isobutyl alcohol, CAS: 78-83-1; A2: acetophenone, CAS: 98-86-2; B2: (1S) - (-) - betapinene, CAS: 18172-67-3; C2: L (-) - carvone, CAS: 6485-40-1; D2: 5-methyl-2-hexanone, CAS: 110-12-3) that were volatilized with nitrogen air (flow rate, $2 \mathrm{~L} / \mathrm{min}$ ) and diluted with ultrapure air (flow rate, $1 \mathrm{~L} / \mathrm{min}$ ). To prevent cross-contamination, separate Teflon tubing lines were used for each odor. These lines converged into a single channel at the bottom of the odor port. In addition, a vacuum located at the top of the odor port provided constant negative pressure to quickly evacuate odor traces with a matched flow rate.

\section{Sequence memory task training}

Naive rats were initially trained in a series of incremental stages over 15-20 weeks. Each rat was trained to poke and hold its nose in an odor port to receive a water reward. The minimum required nose-poke duration started at $50 \mathrm{~ms}$ and was gradually increased (in $15 \mathrm{~ms}$ steps) until a rat reliably held the nose-poke position for $1.2 \mathrm{~s}$ for at least $80 \%$ of the time over three sessions (200-300 nose-pokes per session). The rats were then habituated to odor presentations in the port (odor $A_{1}$ and $A_{2}$, then odor sequences $A_{1} B_{1}$ and $A_{2} B_{2}$ ) and each rat was required to maintain its nose-poke response for $1 \mathrm{~s}$ to receive a reward. The rats were then trained to identify InSeq and OutSeq items. Rats were initially trained on a two-item sequence: they were presented with "AB" and "AA" sequences in equal proportions. The correct response to the first odor was to hold the nose-poke for $1 \mathrm{~s}$ (Odor A was always the first item). For the second odor, rats were required to determine whether the item was InSeq ( $\mathrm{BB}$; hold for $1 \mathrm{~s}$ to receive reward) or OutSeq (AA; withdraw before $1 \mathrm{~s}$ to receive a reward). After reaching criterion on the two-item sequence, the number of items per sequence was increased to three and four in successive stages (criterion: $70 \%$ correct across all individual odor presentations over three sessions). After reaching 
criterion performance on the two four-odor sequences (70\% correct on both InSeq and OutSeq items), rats underwent surgery for cannula implantation.

\section{Cannula system}

A cannula implant system was created using a high-resolution $(56 \mu \mathrm{m})$ stereolithography 3D printer (ProJet 1200; 3D Systems), suitable for chronic head stages. A custom-designed 3D-printed cannula assembly was created using CAD software (Autodesk Inventor Pro Edition) and assembled with guide cannulas (27 gauge; outer diameter $0.41 \mathrm{~mm}$; inner diameter $0.31 \mathrm{~mm}$; Component Supply Company, $\mathrm{FL})$ targeting the PER bilaterally (A/P $-6.0 \mathrm{~mm}, \mathrm{M} / \mathrm{L} \pm 6.8 \mathrm{~mm}, \mathrm{D} / \mathrm{V}-6.0 \mathrm{~mm})$ and a single site aimed at $\mathrm{RE}\left(\right.$ at a $10^{\circ}$ angle to avoid the superior sagittal sinus; $A / P-1.8 \mathrm{~mm}, \mathrm{M} / \mathrm{L}-1.2 \mathrm{~mm}, \mathrm{D} / \mathrm{V}-6.7 \mathrm{~mm}$ ).

\section{AAV9 microsyringe infusions and cannula placement}

Rats were anesthetized with isoflurane (induction 5\%; maintenance: $2 \%-3 \%$ ) mixed with oxygen (800 ml/min) and placed in a stereotaxic apparatus (David Kopf Instruments, Model 900). A protective ophthalmic ointment (Gentak, $0.3 \%$ ) was applied to the eyes and the scalp was sterilized with applications of isopropyl alcohol $\left(70 \%\right.$ in deionized $\left.\mathrm{H}_{2} \mathrm{O}\right)$ followed by Betadine. The incision site was locally anesthetized with Marcaine ${ }^{\circledR}(7.5 \mathrm{mg} / \mathrm{ml}, 0.5 \mathrm{ml}$, s.c.) and the skull was exposed following a fish eye incision. Adjustments were made to ensure bregma and lambda were level $\left( \pm 0.05 \mu \mathrm{m}\right.$ in the D/V plane). Body temperature $\left(35.9-37.5^{\circ} \mathrm{C}\right)$ was monitored and maintained throughout surgery using a rectal thermometer and circulating water heating pad. Ringer's solution with $5 \%$ dextrose was administered to maintain hydration ( $5 \mathrm{ml}$, s.c.), and glycopyrrolate $(0.2 \mathrm{mg} / \mathrm{ml}, 0.5 \mathrm{mg} / \mathrm{kg}$, s.c.) was administered to prevent respiratory difficulties.

Burr holes were drilled bilaterally over mPFC (infusion site; OmniDrill35, World Precision Instruments). Infusions were performed using a $10 \mu \mathrm{L}$ microsyringe (NanoFil; World Precision Instruments) and an infusion pump (UltraMicroPump III; World Precision Instruments). $h M 4 \mathrm{Di}^{+}$rats $(\mathrm{n}=13)$ received $0.5 \mu \mathrm{L}$ injections of the custom AAV-hM4D ${ }_{i}^{\text {nrxn }}\left(A A V 9 . C A G . m C h e r r y-2 a-h M 4 D_{i}{ }^{n r x n}\right.$. WPRE.SV40; UPenn Vector Core) bilaterally into mPFC (A/P $3.24 \mathrm{~mm}, \mathrm{M} / \mathrm{L} \pm 0.7 \mathrm{~mm}, \mathrm{D} / \mathrm{V}$ from cortex $-2.8 \mathrm{~mm})$ at a flow rate of $50 \mathrm{nl} / \mathrm{min}$. mCherry-only rats $(\mathrm{n}=9)$ received $0.5 \mu \mathrm{L}$ injections of the AAV without hM4Di (AAV9.CB7.Cl.mCherry. WPRE.rBG; UPenn Vector Core) bilaterally into mPFC. Pilot experiments were performed to determine the viral gestation time and viral expression. One group of rats $(n=4)$ was injected with AAV9.hM4Di and then perfused at 1 week, 2 weeks, 4 weeks, or 8 weeks. In another set of rats $(n=2)$, saline was injected into mPFC in the left hemisphere and AAV9.hM4Di was injected into mPFC in the right hemisphere to determine the mCherry fluorescence from the virus. The AAV9.CB7.CI.mCherry was injected into three rats at dilutions of 1:2, 1:4, or 1:8 to measure the expression rate compared with the $\mathrm{hM}_{4} \mathrm{Di}^{+}$group to determine the optimal concentration for use in the control group.

Following injection of the viral vector into mPFC, burr holes overlying the PER bilaterally (A/P $-6.0 \mathrm{~mm}, \mathrm{M} / \mathrm{L} \pm 6.8 \mathrm{~mm}, \mathrm{D} / \mathrm{V}$ $-6.0 \mathrm{~mm}$ ) and $\mathrm{RE}$ (at a $10^{\circ}$ angle to avoid the superior sagittal sinus; $\mathrm{A} / \mathrm{P}-1.8 \mathrm{~mm}, \mathrm{M} / \mathrm{L}-1.2 \mathrm{~mm}, \mathrm{D} / \mathrm{V}-6.7 \mathrm{~mm}$ ) were drilled into the skull. The cannula implant was inserted and secured with skull screws (1/8-inch grade 2 (CP) titanium; Allied Titanium Inc.). The head stage was affixed to the surgical screws with dental cement (methyl, methacrylate, Patterson Dental). Dummy cannulas were inserted into the guide cannulas (extending $0.5 \mu \mathrm{m}$ beyond the tip of the guide cannula) to protect against debris entering the cannula and prevent scar tissue from developing and blocking the inserted tip of the cannula. A protective cap was affixed atop the cannula implant to protect against impact and debris. Excess skin was sutured (black silk suture 4-0, with reverse cutting needle $19 \mathrm{~mm}, 1 / 2$ Circle; FEN suture). Neosporin ${ }^{\circledR}$ was applied to the skin surrounding the head stage. At the end of surgery, Flunixin $(50 \mathrm{mg} / \mathrm{ml}, 2.5 \mathrm{mg} / \mathrm{kg}$, s.c.), a nonsteroidal anti-inflammatory analgesic, was administered to the rats. The rats were returned to a clean recovery incubator and monitored until they awoke. One day following surgery, the dummy cannulas were checked, the rats were administered a dose of Flunixin, and Neosporin ${ }^{\circledR}$ was reapplied.

\section{Suppressing mPFC neurons and projections}

According to the protocol published by Roth (2016), clozapine-n-oxide (CNO; Cayman Chemical Company) was dissolved in 0.5\% DMSO in $0.9 \%$ saline $(1.0 \mathrm{mg} / \mathrm{ml})$. The CNO dose was selected on the basis of its behavioral effectiveness and ability to inactivate terminal activity when intracerebrally infused over hM4Di-expressing terminals (Smith et al., 2016). The vehicle (Veh) was a solution of 0.5\% DMSO in $0.9 \%$ saline. For i.p. injections, either CNO or Veh was administered. Behavioral testing was initiated at 30 min post-injection (randomized). For intracerebral infusions, needles were made so the infusion tip extended $1 \mathrm{~mm}$ past the implanted cannula. CNO and Veh were administered over 10min (at a volume of $1 \mu \mathrm{L}$ ) in both RE and PER. The infusion cannula (32 gauge; outer diameter $0.0095 \mathrm{~mm}$; inner diameter $0.005 \mathrm{~mm}$; Component Supply Company) was left in place for an additional $5 \mathrm{~min}$ to allow for drug diffusion. Behavioral testing was initiated 30min post-infusion. Both Veh and CNO infusions in RE and PER were counterbalanced and randomized.

\section{Dual retrograde tracing}

Alexa Fluor 488 Cholera Toxin Subunit B (CTB-488) and Alexa Fluor 594 Cholera Toxin Subunit B (CTB-594; Molecular Probes; Invitrogen Inc) were dissolved in neutral phosphate buffer (0.06M PB) at $1 \%$ (500 mg dissolved in $50 \mathrm{~mL}$ of $0.06 \mathrm{M} \mathrm{PB})$ concentration. The solution was aliquoted and stored in a $-80^{\circ} \mathrm{C}$ freezer.

Glass pipettes (3.5" 3-000-203 G/X; Drummond Scientific) were pulled using a P2000 Laser-Based MicroPipette Puller (Sutter Instruments) with a tip diameter between $80-100$ microns. The tip was examined under the confocal microscope (Olympus FV1200) to 
verify diameter size and check for any internal flaws. Prior to surgery, the tracer was loaded into a glass pipette which was previously backfilled with mineral oil using a Hamilton syringe (Hamilton Company).

Rats were anesthetized and prepped for surgery as described previously $(n=4)$. The skull was exposed with a straight incision. Adjustments were made to ensure the bregma and lambda were level $( \pm 0.05 \mu \mathrm{m}$ in the D/N plane). Burr holes overlying RE (at a $10^{\circ}$ angle to avoid the superior sagittal sinus; $\left.A / P-1.8 \mathrm{~mm}, \mathrm{M} / \mathrm{L}-1.2 \mathrm{~mm}, \mathrm{D} / \mathrm{V}-6.85 \mathrm{~mm}\right)$ and $\mathrm{PER}(\mathrm{A} / \mathrm{P}-6.0 \mathrm{~mm}, \mathrm{M} / \mathrm{L}$ $-7.2 \mathrm{~mm}, \mathrm{D} / \mathrm{V}-6.6 \mathrm{~mm}$ ) were drilled into the skull (OmniDrill35, World Precision Instruments). Infusions were performed using a glass pipette and an infusion pump (Nanoject III, Drummond Scientific). $0.3 \mu \mathrm{l}$ was injected into RE and $0.5 \mu \mathrm{l}$ was injected into PER at a flow rate of $1 \mathrm{nl} / \mathrm{s}$. Three of the rats received CTB-488 in RE and CTB-594 in PER and one rat received the opposite. After the tracer was fully injected, 15mins was allotted to allow for diffusion. Negative pressure was added in order to avoid the spread of the tracer along the injection track. The incision site was sutured (black silk suture 4-0, with reverse cutting needle $19 \mathrm{~mm}, 1 / 2$ Circle; FEN suture) and dressed with Neosporin ${ }^{\circledR}$. At the end of surgery, Flunixin $(50 \mathrm{mg} / \mathrm{ml}, 2.5 \mathrm{mg} / \mathrm{kg}$, s.c.), a nonsteroidal anti-inflammatory analgesic, was administered to the rats. The rats were returned to a clean recovery incubator and monitored until they awoke. One day following surgery the rats were administered a dose of Flunixin and Neosporin ${ }^{\circledR}$ was reapplied. After a 2-week incubation period, the rats were perfused.

\section{Histology}

Rats were anesthetized with isoflurane (5\%) mixed with oxygen (800 ml/min), and transcardially perfused with $100 \mathrm{~mL}$ phosphatebuffered saline, followed by $200 \mathrm{~mL}$ of $4 \%$ paraformaldehyde (PFA, pH 7.4; MilliporeSigma). Brains were post-fixed overnight in 4\% PFA and then placed in a $30 \%$ sucrose solution for cryoprotection. Frozen brains were cut on a sliding microtome (40 $\mu$ m; coronal plane) into three sets of immediately adjacent sections.

To visualize AAV9.hM4Di expression as well as cannula tracts, half the slices in the first set (set 1) were mounted and coverslipped using Vectashield ${ }^{\circledR}$ antifade mounting medium with 4',6-diamidino-2-phenylindole. An mCherry reporter molecule was expressed with hM4Di and visualized with a confocal microscope (Olympus FV1200) using standard filter cubes. The remaining slices in set 1 were mounted for a cell body-specific cresyl violet stain and coverslipped with Permount to visualize the cannula placement. A separate set of slices was processed for immunohistochemistry to visualize the extent of infected mPFC terminals in each of the cannula target sites (RE and PER). Free-floating sections were placed in primary antibody solution: polyclonal rabbit anti red fluorescent protein (Rockland Inc.) in $0.1 \%$ bovine serum albumin (BVA) at a 1:1000 concentration for 24-48h. The sections were then washed with $0.1 \mathrm{M}$ phosphate buffer (PB), placed in a 1:500 dilution of the secondary antibody (biotinylated goat anti-rabbit, Vector Laboratories) for 2h. After washing, the sections were incubated for $1 \mathrm{~h}$ using the avidin biotin complex (ABC) Elite kit (Vector Labs) in the diluent at a 1:300 concentration. Following the final washes, brown cell bodies and fibers expressing mCherry were visualized by incubating the tissue in $0.022 \% 3,3^{\prime}$-diaminobenzidine and $0.003 \%$ hydrogen peroxide for approximately $2-4 \mathrm{~min}$. Sections were then mounted on chromalum gelled slides, dehydrated in graded methanols, and placed in xylene before being coverslipped with Permount.

To map the spread of the injection, coronal micrographs of whole slices across the frontal cortex of tissue processed for the mCherry antisera were obtained at 100x using a NikonFI-3 mounted on a Nikon Eclipse E600 microscope for each rat (Figure S6). The micrographs were transposed over rendered plates drawn from Nissl sections and modified schematic plates (Paxinos and Watson, 2004) in Procreate (Savage Software Group). These images were imported into Adobe Illustrator (Adobe Inc.) where labeled cell bodies were identified and used to pixelate and create a shaded image of the injection spread, such that the shading intensity (pixilation) corresponded to the density of cell expression. The opacity of the shading was reduced to $40 \%$ and images of all rats were superimposed onto one another to create a final schematic at five anterior posterior levels across mPFC.

A series of tissue was processed in a subset of animals $(n=3)$ for dual immunofluorescence using antibodies for mCherry and NeuN to quantify the DREADD transduction rate in $\mathrm{hM} 4 \mathrm{Di}^{+}$rats. For this, free-floating sections were incubated in the primary antibody rabbit anti-red fluorescent protein for 24-48h. Following PB washes, the tissue was placed in an antisera for NeuN, (Mouse Anti-NeuN Antibody, clone A60; EMD Millipore) for 4h. Following another set of PB washes, the tissue was incubated in fluorophore-conjugated secondary antibodies (VectaFluor Duet Immunofluorescence Double Labeling Kit, DyLight 594 Anti-Rabbit/DyLight 488 Anti-Mouse) for an additional $2 \mathrm{~h}$ before being mounted onto chrom-alum gelled slides, rinsed in deionized $\mathrm{H}_{2} \mathrm{O}$, and coverslipped using VECTASHIELD HardSet Antifade Mounting Medium.

For the rats $(n=4)$ with the dual retrograde tracer, the first set were mounted and coverslipped using Vectashield $\AA$ antifade mounting medium with 4',6-diamidino-2-phenylindole. The injection sites and mPFC were visualized with a confocal microscope (Olympus FV1200) using standard filter cubes. To identify mPFC projection cell type, the second set of tissue was processed for immunofluorescence using the antibody for glutamic acid decarboxylase (GAD). Sections were blocked in 0.5\% BSA for one hour then placed in the primary antibody, mouse anti-GAD67 (EMD Millipore Inc) at a concentration of 1:2000 for 36h. Following PB washes, the tissue was incubated in the secondary antibody, Dylight 405 goat anti-mouse (Invitrogen Inc) for $3 \mathrm{~h}$. Following final washing of tissue, sections were mounted and coverslipped with VECTASHIELD $®$ Antifade Mounting (Vector Labs).

\section{QUANTIFICATION AND STATISTICAL ANALYSIS}

All data were analyzed in MATLAB 2016a (Mathworks), SPSS 20.0.0, Excel 2016, and FIJI ImageJ using custom scripts and functions. 
Quantification of mPFC cells and fiber density in RE and PER

For the dual immunofluorescence using antibodies for mCherry and NeuN identical epifluorescent images of the frontal cortex were captured at 100x with a NikonFI-3 camera using NIS Elements software (Nikon) with filters for red fluorescent protein (hM4Di/ mCherry-labeled neurons) and green fluorescent protein (NeuN-labeled neurons) at three separate anterior posterior levels: at the core of the injection ( 3.2 from Bregma), one level anterior, and one level posterior $( \pm 120 \mu \mathrm{m}$ in distance). Images were then imported into FIJI ImageJ (Version 2.0.0; NIH), and a uniform region of interest at each level of the prelimbic cortex and anterior cingulate cortex, restricted to layers 5/6, which expressed the greatest density of hM4Di neurons, was selected to estimate the maximum percentage of cells infected. Manual cell counts were conducted using the Cell Counter plug-in in FIJI and the ratio of mCherry labeled neurons to neurons expressing NeuN was calculated and averaged across rats.

The pattern of hM4Di expression in terminals was analyzed using DAB immunohistochemistry in a subset of rats $(n=3)$ by capturing serial sections across the anterior posterior (AP) plane of RE and PER using at 100x magnification. Photomicrographs were then converted to 8-bit images in FIJI. The mean density was calculated by determining the absolute value of the difference of the mean gray value from the maximum gray area (255) through a ROI for each terminal site (RE or PER). The mean density was then normalized by dividing it over the mean gray density of a reference area consistent across each structure (3V; rhinal fissure) to obtain the relative density (RD) of each ROI. Normalizing the data removed any variability in the intensity of staining across sections and cases. For $\mathrm{RE}, 100 \mathrm{um}^{2} \mathrm{ROls}$ were measured at $6 \mathrm{AP}$ levels for the medial (REm), dorsal (Red) and lateral (REI) division of anterior RE, the medial (REcm), dorsal (REcd) divisions of caudal RE, and perireuniens (periRE). For PER, 50um ${ }^{2}$ ROls were measured at $3 \mathrm{AP}$ levels for each layer of cortex. The RD was then used to conduct a quantitative analysis of the distribution and density of hM4Di ${ }^{+}$fibers from $\mathrm{mPFC}$ to RE and PER.

The number of dual labeled cells (CTB-594, CTB-488) were counted using the methods mentioned above. Images were taken at 20X magnification using a confocal microscope (FV1200) to look for double-labeling across mPFC layers. Cells were counted in FIJI ImageJ (Version 2.0; NIH) for CTB-488 and CTB-594 labeled cells at eight locations (anterior cingulate cortex, dorsal prelimbic cortex, ventral prelimbic cortex, and infralimbic cortex; at $\sim 3.72$ from Bregma and $\sim 3.00$ from Bregma). Individual sections were then separated by layers $(\mathrm{I}, \mathrm{II} / \mathrm{III}, \mathrm{V}, \mathrm{VI})$ and a uniform $\mathrm{ROI}\left(300 \mu \mathrm{m}^{2}\right)$ was taken at each layer of $\mathrm{mPFC}$ to look for double-labeling. Two separate manual cells counts were conducted using the Cell Counter plug-in in FIJI and the ratio of CTB-488 or CTB-594 labeled cells to the total number of DAPI (blue) labeled cells were counted and averaged across animals. For consistency, we processed the colors of the images so $\mathrm{mPFC} \rightarrow \mathrm{RE}$ was always green and $\mathrm{mPFC} \rightarrow \mathrm{PER}$ was always cyan.

For the GAD stained sets, we looked for double-labeling of GAD67 (blue) and CTB-488/594 labeled cells in mPFC. Images were taken at 60X magnification and analyzed in FIJI for any dual-labeled cells.

\section{Statistics}

Performance on the task was analyzed using a number of measures. The first position of each sequence was excluded from all analyses as these items are always InSeq. Expected versus observed frequencies were analyzed with G-tests to determine whether the observed frequency of InSeq and OutSeq responses for a given session significantly differed from the frequency expected by chance. G-tests provide a measure of performance that controls for response bias and is a robust alternative to the $\chi^{2}$ test, especially for datasets that include cells with smaller frequencies (Sokal and Rohlf, 1995). To compare performance across sessions or animals, a sequence memory index was calculated (SMI; Allen et al., 2014) according to the following equation:

$$
\mathrm{SMI}=\frac{\left(0.9 * \mathrm{IN}_{\text {cor }}\right)\left(0.1 * \mathrm{OUT}_{\text {cor }}\right)-\left(0.9 * \mathrm{IN}_{\text {inc }}\right)\left(0.1 * \mathrm{OUT}_{\text {inc }}\right)}{\sqrt{\left(0.9 * \mathrm{IN}_{\text {cor }}+0.9 * \mathrm{IN}_{\text {inc }}\right)\left(0.1 * \mathrm{OUT}_{\text {cor }}+0.1 * \mathrm{OUT}_{\text {inc }}\right) \times\left(0.9 * \mathrm{IN}_{\text {cor }}+0.1 * \mathrm{OUT}_{\text {inc }}\right)\left(0.9 * \mathrm{IN}_{\text {inc }}+0.1 * \mathrm{OUT}_{\text {cor }}\right)}}
$$

The parameters of the equation are as follows: INcor = InSeq correct, INinc = InSeq incorrect, OUTcor = OutSeq correct, and OUTinc = OutSeq incorrect. The SMI normalizes the proportion of InSeq and OutSeq items presented during a session and reduces sequence memory performance to a single value ranging from -1 to 1 . A score of 1 represents perfect sequence memory; i.e., a rat correctly held its nose-poke response to all InSeq items and correctly withdrew its nose on all OutSeq items. A score of 0 indicates chance performance. Negative SMI scores represent performance levels below that expected by chance. A behavioral curve was analyzed for each rat to determine whether it performed above chance levels by measuring the SMI during each session, excluding CNO infusion days. SMI was calculated to determine if the effects of CNO and Veh differed when administered i.p. and by infusion (RE and PER). Using SPSS, an ANOVA was used to analyze the overall effects of Veh versus CNO between repeated conditions of $\mathrm{hM}_{\mathrm{DDi}}{ }^{+}$and mCherry-only groups. In addition, a simple linear regression was used to analyze the relationship between repeated conditions and infusions. Nose-poke duration was analyzed using paired t tests to determine whether rats held their responses significantly longer in $\mathrm{InSeq}_{\text {correct }}$ than in OutSeq $\mathrm{q}_{\text {correct }}$ trials. Paired t tests were performed to determine any differences between the effects of Veh and CNO on inter-odor-interval and inter-sequence-interval. General poke distributions were created through MATLAB using the session data.

Two distinct types of OutSeq probe trials were used to determine sequence memory: backward lags and forward lags. Backward lags occur when an odor is repeated in the sequence (e.g., ABBDD). In this task there were three backward lags (-3-Back, -2-Back, 
and -1-Back) indicating the number of stimuli between the original position and the repeated position. Forward lags occur when the sequence skips ahead (e.g., ADCD). In this particular task, there are two forward lags (+1-Fwd, and +2-Fwd), indicating the number of stimuli between the repeated position and the original position. Lag analyses were used to measure performance on specific forward and backward lag trials for OutSeq items. The accuracy was calculated for each rat. Each individual rat's performance in the Veh condition was subtracted from its performance in the CNO condition for each lag and then divided by the difference in the lag and Veh accuracy. This produced a value that indicated the percent drop in lag performance based on each individual rat's performance in the Veh condition. A paired t test was performed between RE InSeq and PER InSeq performance levels. A one-tailed, onesample $t$ test was performed to measure differences against no change for all lag distances. A repeated-measures $2 \times 3$ ANOVA with a Greenhouse-Geisser correction was performed on the backward lag trials to analyze any interaction effects between RE and PER infusions. A 2x2 ANOVA with a Greenhouse-Geisser correction was performed for the forward lags between RE and PER infusion. The Greenhouse-Geisser was used because the assumption of sphericity is violated for within-subject analysis in most cases and the Greenhouse-Geisser correction is robust to this violation. Finally, $t$ tests and repeated-measures ANOVA in SPSS were conducted for cell counts and fiber densities. 